\title{
Damage Precursor Detection for Structures Subjected to Rotational Base Vibration
}

Ed Habtour and Daniel P. Cole, U. S. Army Research Laboratory, Vehicle Technology

Directorate, APG, MD, 21005 USA

Tel: +01-410-278-8042, Email: ed.m.habtour.civ@mail.mil

Samuel C. Stanton, U. S. Army Research Office, Mechanical Science, APG, MD, 21005 USA

Raman Sridharan and Abhijit Dasgupta, University of Maryland, Center for Advanced Life

\section{Abstract}

Cycle Engineering, College Park, MD, 20742 USA

This paper presents a nonlinear dynamic methodology for monitoring precursors of fatigue damage in metallic structures under variable rotational base excitation. The methodology accounts for important nonlinearities due to the complex loading generated by variable rotation and structural degradation. The sources of the nonlinearities include: structural stiffening due to gyroscopic motion and high-response amplitude at the fundamental mode, softening due to inertial forces and gyroscopic loads, and localized microscopic material damage and microplasticity. The loading intensity and number of vibration cycles increase the influence of these effects. The change in the dynamic response due to fatigue damage accumulation is experimentally investigated by exciting a cantilever beam at variable rotational base motions. The observed fatigue evolution in the material microstructure at regions of large stresses (and the resulting progressive structural softening) is tracked by quantifying the growth in the tip response, the change in the fundamental natural frequency of the beam and the skewedness of the stepped-sine response curve. Previous understanding of the structural dynamic behavior is necessary to ascertain the damage precursor location and evolution. Nanoindentation studies near the beam clamped boundary are conducted to confirm the gradual progression in the local mechanical properties as a function of loading cycles, and microstructural studies are conducted to obtain qualitative preliminary insights into the microstructure evolution. This study demonstrates that careful monitoring of the in the nonlinearities in the structural dynamic 
response can be a sensitive parameter for detection of damage precursors.

Keywords: Damage precursor, fatigue, nonlinear vibration, damage detection, gyroscopic

\section{Introduction}

There are many engineering systems that experience variable rotational base excitations, such as flexible robotics components, heavy electronics components, and unmanned ground and aerial systems $[1,2]$. Many of these systems may experience harsh dynamic loads during their life cycles $[3,4]$. Mechanical structures under variable rotational base excitations may experience complex dynamic effects, such as gyroscopic stiffening and softening, nonlinear geometric stiffening, and nonlinear inertial softening $[1,2]$. Modeling and measuring the dynamic response can be complicated when the material properties evolve due to fatigue damage accumulation. In this study, we focus on the growth of early precursors to such fatigue degradation. Fatigue damage precursor is defined as any observable early degradation of the material microstructural properties prior to crack initiation [5]. Precursors to fatigue crack development may involve, but are not restricted to, changes in the microstructure, chemical composition, electrical signal, acoustic response, or thermal signature of a structure.

This study demonstrates that the dynamic response of a structure due to rotational vibration excitation can serve as a particularly sensitive precursor for fatigue degradation evolution. To facilitate this demonstration, an analytical nonlinear dynamic model is provided, which includes multiple sources of nonlinearities due to the variable harmonic rotational base excitation and the high amplitude response. The presented methodology shows that it is possible to capture fatigue damage precursors by simply tracking the change in the nonlinear stiffness term in the equation of motion. The nonlinear equation of motion is then updated accordingly to capture the local microstructural evolution by adjusting the nonlinear structural stiffness term. The global 
nonlinear vibration-based method uses the nonlinear structural updates from the experiments to estimate the beam tip response and number of fatigue cycles. The technique can be used to assess the structural durability of mechanical systems when they are exposed to complex vibratory loading using conventional sensors, such as accelerometers. Therefore, it is possible to utilize the proposed methodology to detect fatigue damage precursors in mechanical components using current health monitoring systems (SHM) by including the nonlinear terms in their detection codes; this can be done without physical replacement or the addition of sensors.

Multiple studies have been devoted to nonlinear dynamics and vibrations of beams because of their importance in many engineering applications. One of the most cited studies in nonlinear dynamics is the effort presented by Hodges and Dowell [6], where they developed the equations of motion for a rotor blade idealized as a beam, using Hamilton's principle. They maintained the cubic nonlinear terms and included the effect of warping. Partial-differential equations for the motion of nonlinear inextensional and extensional beams were developed for planar and nonplanar base excitation, where the geometric and inertial nonlinearities were included [7-12]. Beam models were improved to idealize more complex structures such as antenna and radar structures, aircraft wings, and wind turbines. Some of these modeling improvements included stepped beams and L-shape beams with tip masses [13-17].

Several researchers have investigated the dynamics of rotating linear and nonlinear flexible beams and flexible hub-beams (rotor-blades) with tip mass [18-20]. These dynamic models were developed in a consistent manner through the formulation of energy expressions and application of Hamilton's principle. The models included dynamic stiffening and inertial softening [21]. Hodges [22] developed nonlinear dynamic models for composite beams and blades exposed to constant base rotational velocity. Radially rotating uniform linear beams under constant and non- 
constant angular velocity has also been investigated [23-25]. However, most of the research performed in studying the vibrational behavior of radially rotating beams has focused on constant spin velocity with various combinations of free, clamped, and simply-supported boundaries $[17,20,26]$.

Absent in the structural dynamics and structural health monitoring literature is a comprehensive theoretical and experimental study of the response of structures exposed to vibrational rotational base excitation that includes all of the following sources of nonlinearities: 1) geometric stiffening, 2) inertial effect, 3) gyroscopic stiffening and softening, 4) higher order nonlinear gyroscopic stiffening, and 5) coupling between the rotational excitation (primary motion) and local displacement (secondary motion). These nonlinearities may potentially become significant when modeling fast and flexible robot manipulators, robotic arms, and adaptive structures under nonlinear oscillatory motion. High cycle vibrations in these structures may lead to fatigue, instability, and loss of position accuracy. The problem can be exacerbated in structures where the system dynamic response progressively changes as the material continues to degrade due to the accumulation of cyclic fatigue damage.

In the current investigation, we exploit the sensitivity of the geometric stiffness to the accumulation of fatigue damage by modifying the nonlinear equation of motion to account for degradation in the local stiffness at high-stress sites. The approach appears to be a promising metric for providing sensitive and robust structural health monitoring to predict fatigue damage precursor in metallic structures. The study shows that the nonlinear vibration-based measurement techniques sense the development of fatigue damage precursors prior to crack initiation. The fatigue-induced structural softening of the structure was evident even at an excitation level where the global deformation remained in the elastic domain. Scanning Electron Microscopy (SEM) 
observations confirmed changes in the material microstructure at high stress concentration regions with the accumulation of fatigue cycles. Nanoindentation measurements at the regions of high stress concentration revealed an accompanying reduction in the apparent stiffness (nanoindentation stiffness) of the material. This study demonstrates the effectiveness of nonlinear vibration response to identify fatigue damage precursors in slender structures through the application of global damage detection methods.

\section{Modeling Development}

The focus of this paper is to examine the nonlinear response of a slender isotropic cantilever beam exposed to harmonic rotation base excitation near its fundamental frequency and the influence of fatigue cycles. The authors studied a cantilever beam with an aspect ratio of 8 (AR=length/width); a detailed discussion is provided in Section 3. The experimental results demonstrated progressive structural softening due to forward-stepped harmonic rotation excitations with extended dwells near the fundamental natural frequency. An analytical model is developed using nonlinear Euler-Bernoulli beam theory with a tunable nonlinear stiffness term to account for observed changes in the global structural response due to possible degradations in the local material stiffness near the beam root (at the fixed end). In other words, the model uses a global structural parameter to capture the global structural softening response due to local changes in the material microstructure. The model also accounts for additional sources of nonlinearities, which include: inertial effects, gyroscopic stiffening and softening, high order nonlinear gyroscopic stiffening, and coupling between the rotational excitation and the corresponding local displacement.

\subsection{Kinematics Development:}

Figure 1 illustrates the dynamics of a cantilever beam with a uniform cross-section carrying a tip 
mass exposed to a variable rotational base excitation. The beam is idealized as an inextensional beam; that is, stretching of the neutral axis is insignificant [27]. The beam is fixed rigidly at one end and left free at the other end (Figure 1). Nonlinear Euler-Bernoulli theory is employed to estimate the beam tip displacement, where the effects of warping and shear deformation are neglected [14].
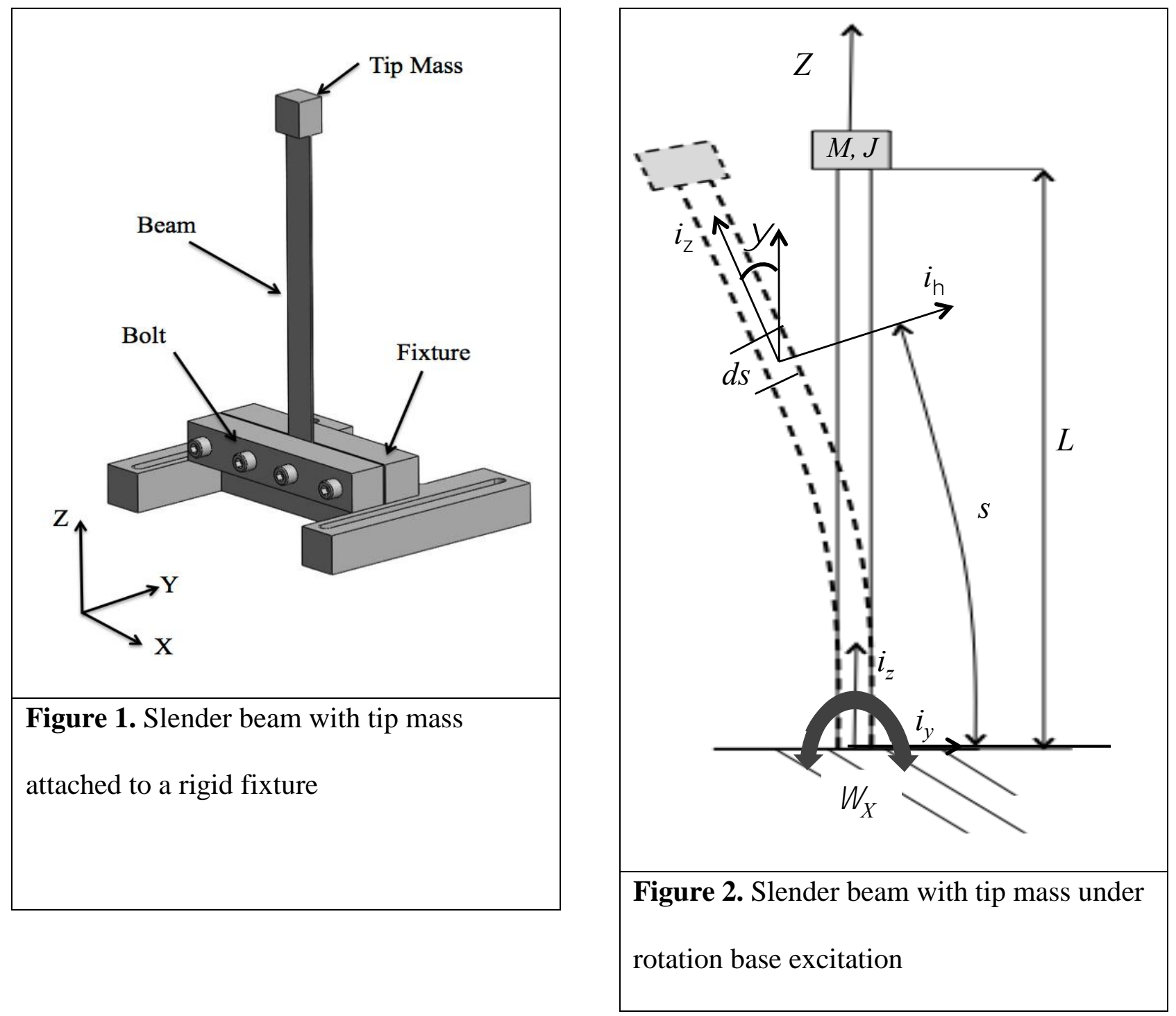

At the large peak response amplitudes, the nonlinear terms in the equations of motion become as important as the linear ones. The beam length-to-width ratio is long enough to cause significant nonlinear flexural deformation but is short enough to assume that the beam undergoes purely 
planar flexural vibrations as long as the tip mass and cross-section geometry are symmetric with respect to the beam centerline [5]. The first flexural mode is approximated to be the same as the mode shape generated from solving the linear problem with tip mass that contains the rotary inertia effect.

The cantilever beam is considered to be a uniform and straight isotropic structure of length $L$ and volumetric density $\varrho$, and clamped at the base with a tip mass $M$, and a rotary inertia $J$, as shown in Figure 2. The $Z$-axis is taken as the neutral axis associated with pure bending. It is assumed the loci of both shear centers and mass centers of the undeformed beam are coincident with the $Z$-axis. The base motion causes each cross section of the beam to experience an elastic displacement of its centroid. The beam dynamic response with respect to the $Y$ and $Z$ axes at the undeformed length from the root of the beam to the reference point, $s$, and time, $t$, is described in terms of: the axial displacement, $w(s, t)$, the transverse displacement $v(s, t)$ along the inertial coordinate system $Y Z$, and the rotational angle $\psi$. The orthogonal unit vectors for the inertial coordinate system are $\left(i_{Y}, i_{Z}\right)$. The local curvilinear coordinate system at $s$, in the deformed position, has the orthogonal unit vectors $\left(i_{\eta}, i_{\zeta}\right)$. The rotating reference frame approach is used, where the reference frame is attached to the undeformed position of the beam (at the base of the beam). The position vector of a point is expressed as follows:

$$
\boldsymbol{R}=v \boldsymbol{i}_{\boldsymbol{Y}}+(s+w) \boldsymbol{i}_{\boldsymbol{Z}}+\eta \boldsymbol{i}_{\eta}
$$

The variable $s$ denotes the arc-length along $\boldsymbol{R}$, and the local deformation is $\eta i_{\eta}$. Differentiating $\boldsymbol{R}$ with respect to time, $t$, yields:

$$
\dot{\boldsymbol{R}}=(\dot{v}-\eta \dot{\psi} \sin (\psi)) \boldsymbol{i}_{Y}+(\dot{w}+\eta \dot{\psi} \cos \psi) \boldsymbol{i}_{Z}+\boldsymbol{\Omega}_{\text {Base }} \times \boldsymbol{R}
$$

where:

$$
\boldsymbol{\Omega}_{\text {Base }}=\Omega_{x}(t) \boldsymbol{i}_{\boldsymbol{x}}
$$


The tracking frame primary motion is $\Omega_{x}(t)$. Since the reference frame is selected a priori, $\Omega_{x}(t)$ becomes a known quantity instead of a variable [28]. Substituting $\boldsymbol{\Omega}_{\text {Base }} \times \boldsymbol{R}$ and combining terms yields the following:

$$
\begin{aligned}
\dot{\boldsymbol{R}}=(\dot{v}-\eta \dot{\psi} & \left.\sin (\psi)-s \Omega_{X}-w \Omega_{X}-\eta \Omega_{X} \sin (\psi)\right) \boldsymbol{i}_{Y} \\
& +\left(\dot{w}+\eta \dot{\psi} \cos \psi+v \Omega_{X}+\eta \Omega_{X} \cos (\psi)\right) \boldsymbol{i}_{Z}
\end{aligned}
$$

Eq. 3

\subsection{Equation of Motion Development}

Kinetic energy can be expressed as follows:

$$
T=\frac{1}{2} \int_{0}^{L} \int_{A_{1}}^{A_{2}} \varrho \dot{\boldsymbol{R}} \cdot \dot{\boldsymbol{R}} d A d s+\left.\frac{1}{2} M(\dot{\boldsymbol{R}} \cdot \dot{\boldsymbol{R}})\right|_{s=L}
$$

Eq. 4

Substituting $\dot{\boldsymbol{R}}$ into the kinetic energy expression and set:

$$
\rho=\int_{A_{1}}^{A_{2}} \varrho d A J_{1}=\int_{A_{1}}^{A_{2}} \varrho \eta d A J_{2}=\int_{A_{1}}^{A_{2}} \varrho \eta^{2} d A
$$

Since the reference point coincides with the beam mass centroid and $\eta$ is a principal axis of the differential beam element, $J_{1}$ is set equal to zero. However, the beam rotary inertia is maintained in the kinetic energy. Therefore,

$$
\begin{aligned}
T=\frac{1}{2} \int_{0}^{L}\left[\rho \left(\dot{v}^{2}\right.\right. & \left.+\dot{w}^{2}+v^{2} \Omega_{X}^{2}+(w+s)^{2} \Omega_{X}^{2}-2(s+w) \dot{v} \Omega_{X}+2 v \dot{w} \Omega_{X}\right) \\
& \left.+J_{2}\left(\Omega_{X}+\dot{\psi}\right)^{2}\right] d s \\
& +\frac{1}{2} M\left[\dot{v}^{2}+\dot{w}^{2}+v^{2} \Omega_{X}^{2}+(w+s)^{2} \Omega_{X}^{2}-2(s+w) \dot{v} \Omega_{X}\right. \\
& \left.+2 v \dot{w} \Omega_{X}\right]\left.\right|_{s=L}+\left.\frac{1}{2} J\left(\Omega_{X}+\dot{\psi}\right)^{2}\right|_{s=L}
\end{aligned}
$$

Performing Taylor's expansion up to cubic nonlinearities and assuming that $w$ and $v$ are small but finite, the potential energy and the kinetic energy can be expressed as follows [11]: 


$$
\begin{aligned}
& \Pi=\frac{E I}{2} \int_{0}^{L}\left(v^{\prime \prime 2}+v^{\prime \prime 2} v^{\prime 2}\right) d s-\frac{1}{2} \rho g \int_{0}^{L}(L-s) v^{\prime 2} d s-\frac{1}{2} M g \int_{0}^{L} v^{\prime 2} d s \quad \text { Eq. } 6 \\
& T=\frac{1}{2} \int_{0}^{L}\left[\rho \left(\dot{v}^{2}+\frac{1}{4}\left(\frac{\partial}{\partial t} \int_{0}^{\zeta} v^{\prime 2} d s\right)^{2}+v^{2} \Omega_{X}^{2}\right.\right. \\
& +\left(-\frac{1}{4}\left(\int_{0}^{\zeta} v^{\prime 2} d s\right)^{2}+s \int_{0}^{\zeta} v^{\prime 2} d s+s^{2}\right) \Omega_{X}^{2} \\
& \left.-\left(2 s-\int_{0}^{\zeta} v^{\prime 2} d s\right) \dot{v} \Omega_{X}-v \Omega_{X} \frac{\partial}{\partial t}\left(\int_{0}^{\zeta} v^{\prime 2} d s\right)\right) \\
& \left.+J_{2}\left(\Omega_{X}^{2}+2 \Omega_{X} \dot{v}^{\prime}+\Omega_{X} \dot{v}^{\prime} v^{\prime 2}+\dot{v}^{\prime 2}+\dot{v}^{\prime 2} v^{\prime 2}\right)\right] d s \\
& +\left.\frac{1}{2} M\left(\dot{v}^{2}+\frac{1}{4}\left(\frac{\partial}{\partial t} \int_{0}^{\zeta} v^{\prime 2} d s\right)^{2}\right)\right|_{s=L}+\left.\frac{1}{2} J\left(\dot{v}^{\prime 2}+\dot{v}^{\prime 2} v^{\prime 2}\right)\right|_{S=L}
\end{aligned}
$$

Here, the dots and the primes denote the temporal and spatial derivatives, respectively.

The assumed modes method is used, as it is convenient to implement and is applicable to nonlinear systems [28]. The approximate solutions are assumed in series form, as follows:

$$
v(t, z)=\sum_{j=1}^{N} q_{j}(t) Y_{j}(z)
$$

Eq. 8

where, $q_{j}$ denotes the generalized modal coordinates and $Y_{j}$ is the mass normalized eigenfunction of the $j^{\text {th }}$ free undamped vibration mode:

$$
Y(x)=A_{j}\left[\cos \beta_{j} x-\cosh \beta_{j} x+C_{j}\left(\sin \beta_{j} x-\sinh \beta_{j} x\right)\right] \quad \text { Eq. } 9
$$

where,

$$
C_{j}=\frac{\sin \beta_{j} L-\sinh \beta_{j} L+\beta_{j} L \bar{M}\left(\cos \beta_{j} L-\cosh \beta_{j} L\right)}{\cos \beta_{j} L+\cosh \beta_{j} L-\beta_{j} L \bar{M}\left(\sin \beta_{j} L-\sinh \beta_{j} L\right)}
$$


$A_{j}$ is a modal amplitude constant which is evaluated by normalizing the eigenfunctions, $\beta_{j}$ is the system eigenvalue for mode $j$, and $\bar{M}$ is the normalized tip mass [29]. The eigenfunctions are normalized according to the orthogonality conditions, which include the rotary inertia of the tip mass. However, the main concern in this study is the first mode (flexural mode); thus, a converged solution is achieved with admissible functions that "resemble" the eigenfunctions of a cantilever beam with the same boundary conditions $[28,30]$. Indeed, for the first-mode nonlinear cantilever beam vibration problem, Hamdan, Elvin and Elvin, and Leadenham and Erturk have observed the convergence of the solution with the resembled admissible functions as long as the base displacement is small relative to the tip displacement [30-32]. For single mode, the assumed solution becomes:

$$
v(t, z)=q(t) Y(z)
$$

Error! Reference source not found. is substituted into the kinetic energy and potential energy equations (Eq. 6 and 7). The Euler-Lagrangian equation is then applied to the Lagrangian, $L=T-\Pi$ as follows [28]:

$$
\frac{\partial}{\partial t}\left(\frac{\partial L}{\partial \dot{q}}\right)-\frac{\partial L}{\partial q}=0
$$

The equation of motion with the appropriate nonlinear terms for the system understudy becomes:

$$
a_{1} \ddot{q}+a_{2}\left(q^{2} \ddot{q}+q \dot{q}^{2}\right)+a_{3} \dot{\Omega}_{X} q^{2}+\left(k_{1}-h_{1} \Omega_{X}^{2}\right) q+\left(k_{2}-h_{2} \Omega_{X}^{2}\right) q^{3}=a_{4} \dot{\Omega}_{X} \quad \text { Eq. } 11
$$

Adding a viscous damping term to the equation of motion leads to the final form of the governing equation for a cantilever beam with variable rotational base excitation:

$$
\begin{aligned}
a_{1} \ddot{q}+a_{2}\left(q^{2} \ddot{q}+q \dot{q}^{2}\right)+c \dot{q}+a_{3} \dot{\Omega}_{X} q^{2}+\left(k_{1}-h_{1} \Omega_{X}^{2}\right) q+\left(k_{2}-h_{2} \Omega_{X}^{2}\right) q^{3} \\
=a_{4} \dot{\Omega}_{X}
\end{aligned}
$$

The inertial coefficient including the rotary inertia (or effective mass) is: 


$$
a_{1}=\rho \int_{0}^{L} Y^{2} d s+J_{2} \int_{0}^{L} Y^{\prime 2} d s+\left.M Y^{2}\right|_{s=L}+\left.J Y^{\prime 2}\right|_{s=L}
$$

The nonlinear inertial coefficient including tip rotary inertia is:

$$
\begin{aligned}
& a_{2}=\rho \int_{0}^{L}\left(\int_{0}^{\zeta} Y^{\prime 2} d s\right)^{2} d s+J_{2} \int_{0}^{L} Y^{\prime 4} d s+\left.M\left(\int_{0}^{\zeta} Y^{\prime 2} d s\right)^{2}\right|_{s=L}+\left.J Y^{\prime 4}\right|_{s=L} \\
& a_{3}=\frac{1}{2}\left(-\rho \int_{0}^{L} Y \int_{0}^{\zeta} Y^{\prime 2} d s d s+J_{2} \int_{0}^{L} Y^{\prime 3} d s-\left.M Y \int_{0}^{\zeta} Y^{\prime 2} d s\right|_{s=L}+\left.J Y^{\prime 3}\right|_{s=L}\right)
\end{aligned}
$$

The linear stiffness coefficient (or effective elastic stiffness) is:

$$
k_{1}=E I \int_{0}^{L} Y^{\prime \prime 2} d s-\rho g\left(\int_{0}^{L}(L-s) Y^{\prime 2} d s\right)-\left.M g\left(\int_{0}^{L} Y^{\prime 2} d s\right)\right|_{s=L}
$$

Eq. 16

The gyroscopic stiffening and softening coefficient is [26, 29]:

$$
h_{1}=\rho \int_{0}^{L} Y^{2} d s-\frac{1}{2} \rho \int_{0}^{L}\left(L^{2}-s^{2}\right) Y^{\prime 2} d s+\left.M Y^{2}\right|_{s=L}-\left.M L \int_{0}^{\zeta} Y^{\prime 2} d s\right|_{s=L}
$$

The nonlinear geometric stiffness coefficient is:

$$
k_{2}=2 E I \int_{0}^{L} Y^{\prime \prime 2} Y^{\prime 2} d s
$$

The nonlinear gyroscopic stiffness coefficient is:

$$
h_{2}=\frac{1}{2}\left[\rho \int_{0}^{L}\left(\int_{0}^{\zeta} Y^{\prime 2} d s\right)^{2} d s+\left.M\left(\int_{0}^{\zeta} Y^{\prime 2} d s\right)^{2}\right|_{s=L}\right]
$$

The base excitation inertial coefficient is:

$$
a_{4}=\rho \int_{0}^{L} s Y d s-J_{2} \int_{0}^{L} Y^{\prime} d s+\left.M L Y\right|_{s=L}-\left.J Y^{\prime}\right|_{s=L}
$$

If the rotational base motion is dropped, the equation of motion becomes:

$$
a_{1} \ddot{q}+a_{2}\left(q^{2} \ddot{q}+q \dot{q}^{2}\right)+k_{1} q+k_{2} q^{3}=0
$$


The above equation is the classical free vibration nonlinear equation of motion with the nonlinear inertial effect (terms two and three) and the nonlinear geometric stiffness [33]. However, the variable rotational motion introduces additional sources of nonlinearities as well as augmenting the coupling between the base motion and the local motion. Accordingly, coefficients $h_{1}$ and $h_{2}$ are maintained in the equation of motion (Eq. 12). The contribution of positive terms in $h_{1}$ increases the gyroscopic stiffness while the negative terms augment the gyroscopic softening. The equation of motion produced in this study is an expansion of the rotating beam model provided by Smith and Baruh [26], which did not include the nonlinear geometric stiffness and nonlinear inertial terms:

$$
a_{1} \ddot{q}+\left(k_{1}-h_{1} \Omega_{X}^{2}\right) q=a_{4} \dot{\Omega}_{X}
$$

For flexible structures such as compliant robotic arms and legs, the nonlinear inertial and geometric stiffness effects become important. Furthermore, high displacement vibratory loads may intensify the structural softening effect due to localized fatigue damage precursors at highstress concentration sites. The structural softening effect due to fatigue is globally accounted for by introducing the nonlinear adjustment factor, which is discussed in Section 4 . The contribution of the rotational motion is also included in the nonlinear stiffness calculation (coefficient $h_{2}$ ).

\section{Experimental Approach}

To produce variable rotational base excitations, a multiaxial electrodynamic shaker (Tensor-900 by Team Corporation) was utilized. It consists of eight plane actuators and four out-of-plane actuators underneath the shaker (Figure 3), which are mechanically coupled to the table. The unique architecture of the shaker system allows a true six degrees-of-freedom (DoF) vibration environment (three translations and three rotations). The four out-of-plane actuators underneath the shaker table are employed to drive the base rotation about the $\mathrm{x}$-axis. The other eight 
actuators are used to ensure a pure rotational excitation by reducing potential noise and misalignments. For additional details related to the multiaxial shaker, refer to Ernst et al. [2].
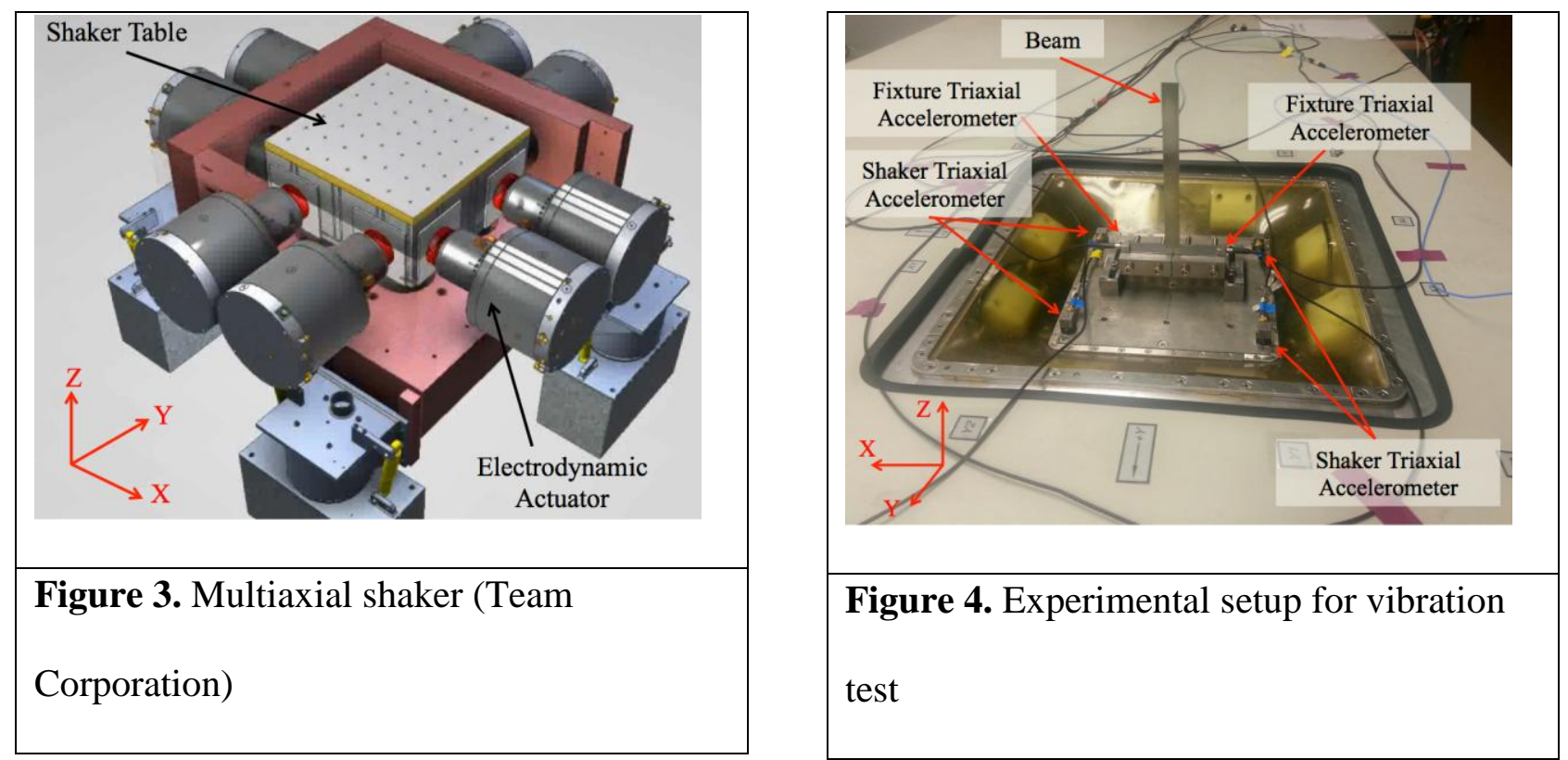

Slender blue-finished and polished spring-tempered AISI 1095 high carbon steel cantilever beams were used in this study. This type of steel is typically fabricated using cold rolling, which may produce some level of material orthotropy. Accordingly, the isotropy assumption is an approximation in this study. The density and the elastic modulus of the material are $7.85 \mathrm{~g} / \mathrm{cm}^{3}$ and $205 \mathrm{GPa}$, respectively. The hardness is Rockwell C48 with $\mathrm{AR}=8$. The beam length and cross-section area are $127 \mathrm{~mm}$ and $15.88 \times 1.08 \mathrm{~mm}^{2}$, respectively.

The vibration experiment setup and instrumentation are shown in Figure 4. Each beam was mounted vertically on a rigid fixture. The torque for each bolt in the fixture was maintained at 22.6 N-m for each test. The multiaxial shaker table was controlled by four Dytran triaxial accelerometers (Model No. 3243M2), with one accelerometer at each corner on the shaker table. Two additional accelerometers were mounted on the fixture (Figure 4) as a backup to ensure that the base excitation was purely rotational. The beam tip displacement was measured using two PCB Piezotronics accelerometers (Model No. 356A01), which were mounted on each side of the 
beam. The mass of each accelerometer was approximately $1.5 \mathrm{~g}$.

Table 1. Nonlinear experimental cases

\begin{tabular}{|c|c|c|c|}
\hline Case & Dynamic Parameters & Test & $\begin{array}{c}\text { Total Cycles } \\
\left(10^{3}\right)\end{array}$ \\
\hline \multirow{4}{*}{ I } & \multirow{4}{*}{$\begin{array}{l}\text { Base rotational acceleration }=30 \mathrm{rad} / \mathrm{s}^{2} \\
\text { Damped natural freq. }=42.35 \mathrm{~Hz}\end{array}$} & 1 & $0-67$ \\
\hline & & 2 & $67-135$ \\
\hline & & 3 & $135-202$ \\
\hline & & 4 & $202-269$ \\
\hline \multirow{5}{*}{ II } & \multirow{5}{*}{$\begin{array}{l}\text { Base rotational acceleration }=50 \mathrm{rad} / \mathrm{s}^{2} \\
\text { Damped natural freq. }=42.35 \mathrm{~Hz}\end{array}$} & 1 & $0-81$ \\
\hline & & 2 & $81-151$ \\
\hline & & 3 & $151-220$ \\
\hline & & 4 & $220-290$ \\
\hline & & 5 & $290-359$ \\
\hline \multirow{4}{*}{ III } & \multirow{4}{*}{$\begin{array}{l}\text { Base rotational acceleration }=70 \mathrm{rad} / \mathrm{s}^{2} \\
\text { Damped natural freq. }=42.33 \mathrm{~Hz}\end{array}$} & 1 & $0-81$ \\
\hline & & 2 & $81-151$ \\
\hline & & 3 & $151-224$ \\
\hline & & 4 & $224-294$ \\
\hline
\end{tabular}

Three sets of experiments (Cases I, II, and III) were conducted. The base excitations for Case I, II and III were maintained at constant rotational amplitudes at 30, 50 and $70 \mathrm{rad} / \mathrm{s}^{2}$, respectively. The details of each case are listed in Table 1. The fundamental natural frequency was measured for each case prior to conducting the tests, which was reported in Table I. Once the damped natural frequency for each beam was identified using sine-sweep excitation, the beams were 
subjected to the rotational harmonic base excitation at discrete forward dwell frequencies near the fundamental frequencies. The ramp-up time and dwell time for each frequency were $30 \mathrm{~s}$ and 25 s, respectively. The ramp-up time and dwell time at each excitation frequency ensured that steady state response conditions were met. The frequency step for each dwell was $0.05 \mathrm{~Hz}$. Therefore, the excitation frequency was increased by $0.05 \mathrm{~Hz}$ every $55 \mathrm{~s}$ ( $30 \mathrm{~s}$ ramp-up plus $25 \mathrm{~s}$ dwell). The loading cycles depended on the shift in the resonance frequency due to material softening, meaning the tests were repeated until significant change in the resonance was observed, as shown in Figure 5 (Case I). The loading cycles range for each test was reported in Table I. The same procedure was followed for Case II and III as shown in Table I and Figures 6 and 7, respectively. The results for Case I, II, and III are discussed in detail in Section 4.

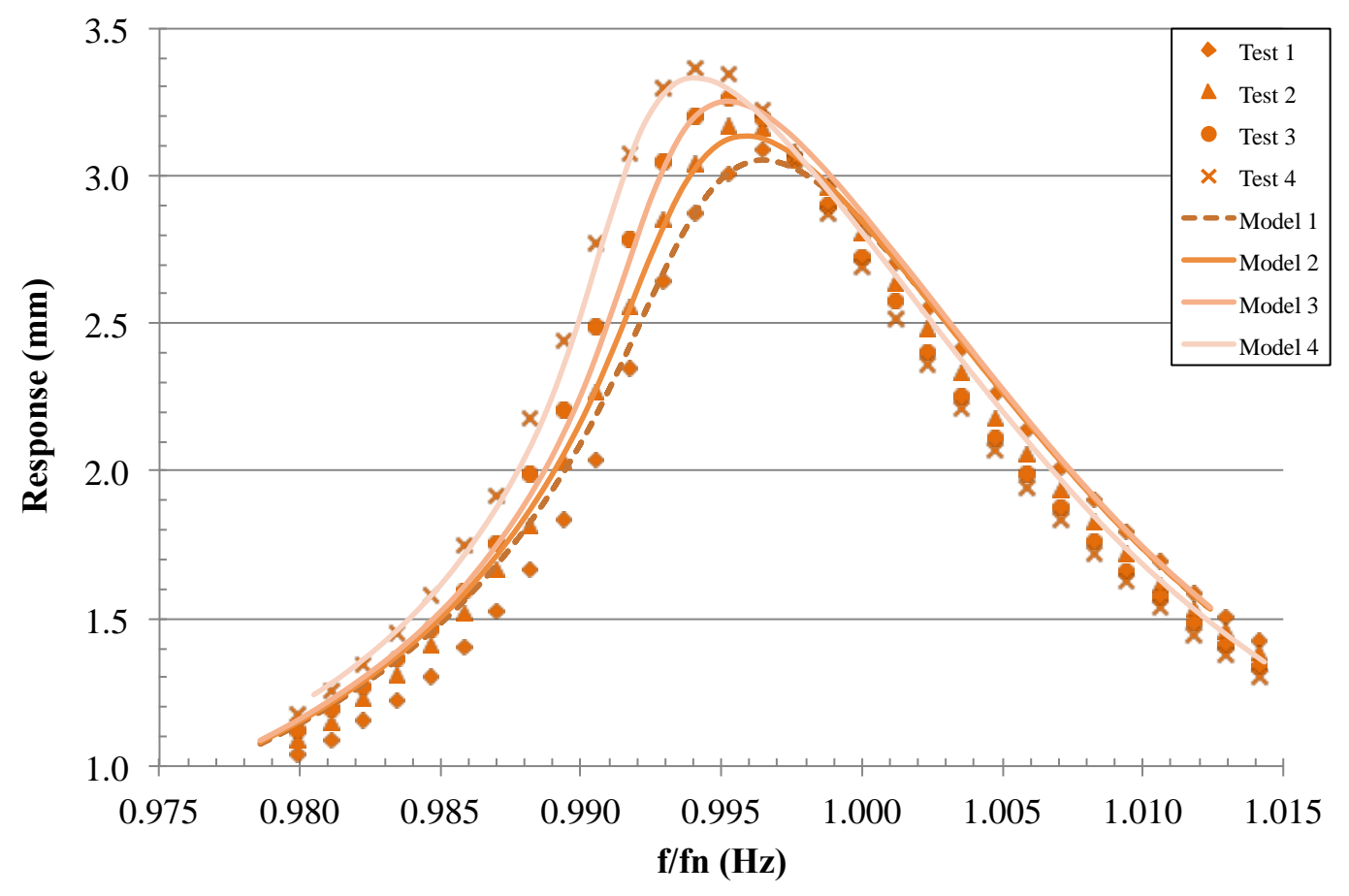

Figure 5. Experimental (markers) and analytical (solid-lines) nonlinear response for Case I Local stiffness properties of the beams used in this study were examined using instrumented indentation [34]. The technique has been frequently used to characterize a wide range of 
complex materials and structures, including film-substrate systems [35], functionally graded structures [36, 37], single microfibers [38, 39], and polymer nanocomposites [40, 41]. In the current study, local mechanical properties of similarly-fatigued beams were probed via instrumented indentation. Samples were polished to $0.1 \mu \mathrm{m}$ and indentation experiments were performed using a Hysitron TI-950 TriboIndenter with a diamond Berkovich tip (radius of curvature approximately $100 \mathrm{~nm}$ ). Positions along the beam surface were located using the optical microscope in the TriboIndenter. For each targeted location, 25 indentations were performed in a $5 \times 5$ grid, spaced $5 \mu \mathrm{m}$ apart. The edge of the initial indent array was positioned approximately $10 \mu \mathrm{m}$ from the clamped edge of the beam; subsequent arrays were positioned in approximately $2 \mathrm{~mm}$ increments stepping away from the clamped boundary position and moving toward the beam-free end. The measurements were performed in a load control mode, with a maximum applied force of approximately $5000 \mu \mathrm{N}$. The applied forces resulted in indentation depths of approximately $150-200 \mathrm{~nm}$ (approximately $0.02 \%$ of the cross-sectional depth). A drift correction was implemented prior to_indentation using $1 \mu \mathrm{N}$ preload for 20s. A triangular force profile was used with a quasi-static loading rate of $625 \mu \mathrm{N} \mathrm{s}^{-1}$. The indentation results provided a reduced elastic modulus $E_{r}$, which is related to the sample elastic modulus $E_{s}$ through: $1 / E_{r}=$ $\left(1-v_{i}^{2}\right) / E_{i}+\left(1-v_{s}^{2}\right) / E_{s}$, where $E_{i}$ is the elastic modulus of the tip and $v_{i}$ and $v_{s}$ are the Poisson's ratio of the tip and sample, respectively. To obtain sample properties, $E_{i}, v_{i}$ and $v_{s}$ were assumed to be $1140 \mathrm{GPa}, 0.07$, and 0.3 , respectively [32]. 


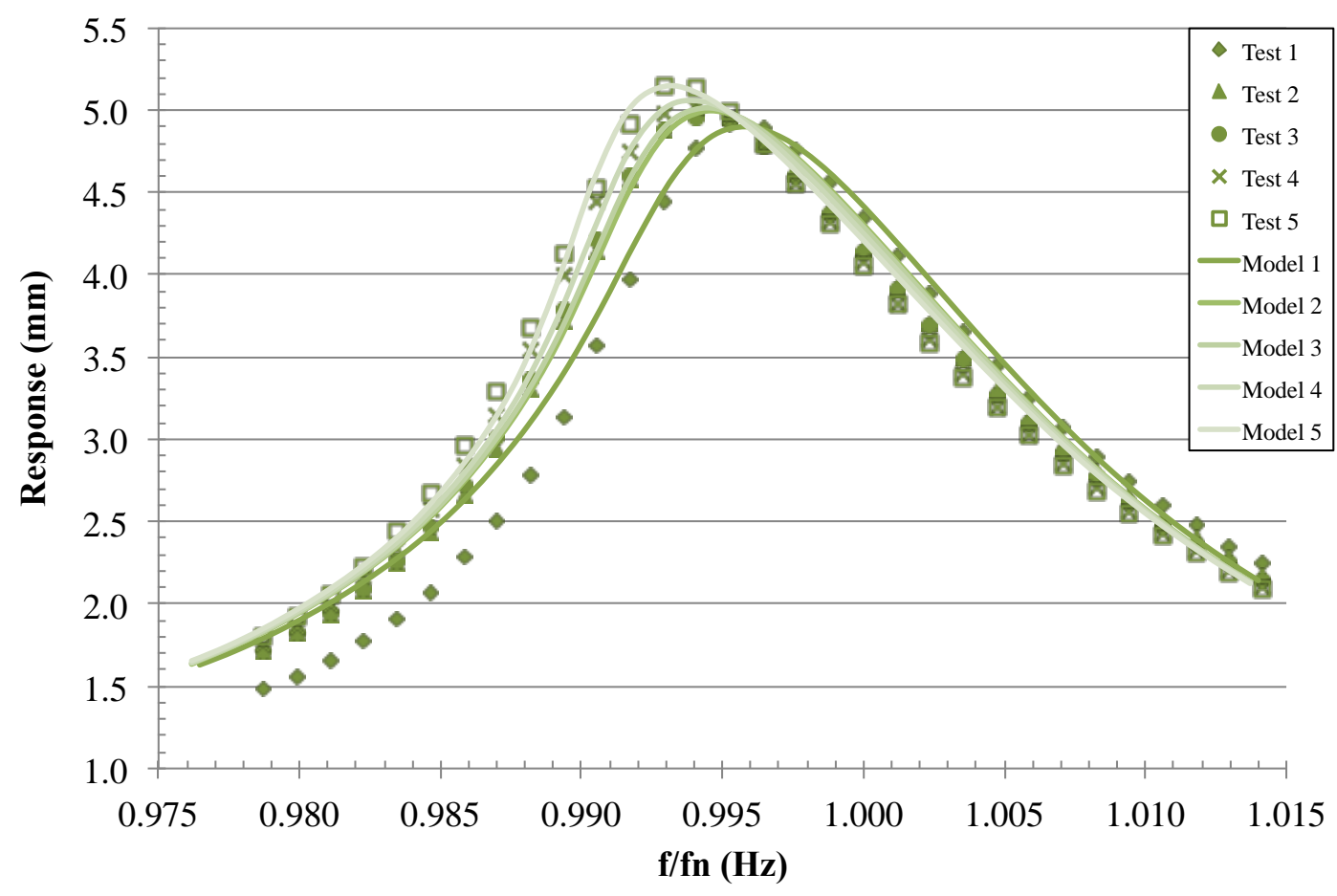

Figure 6. Experimental (markers) and analytical (solid-lines) nonlinear response for Case II

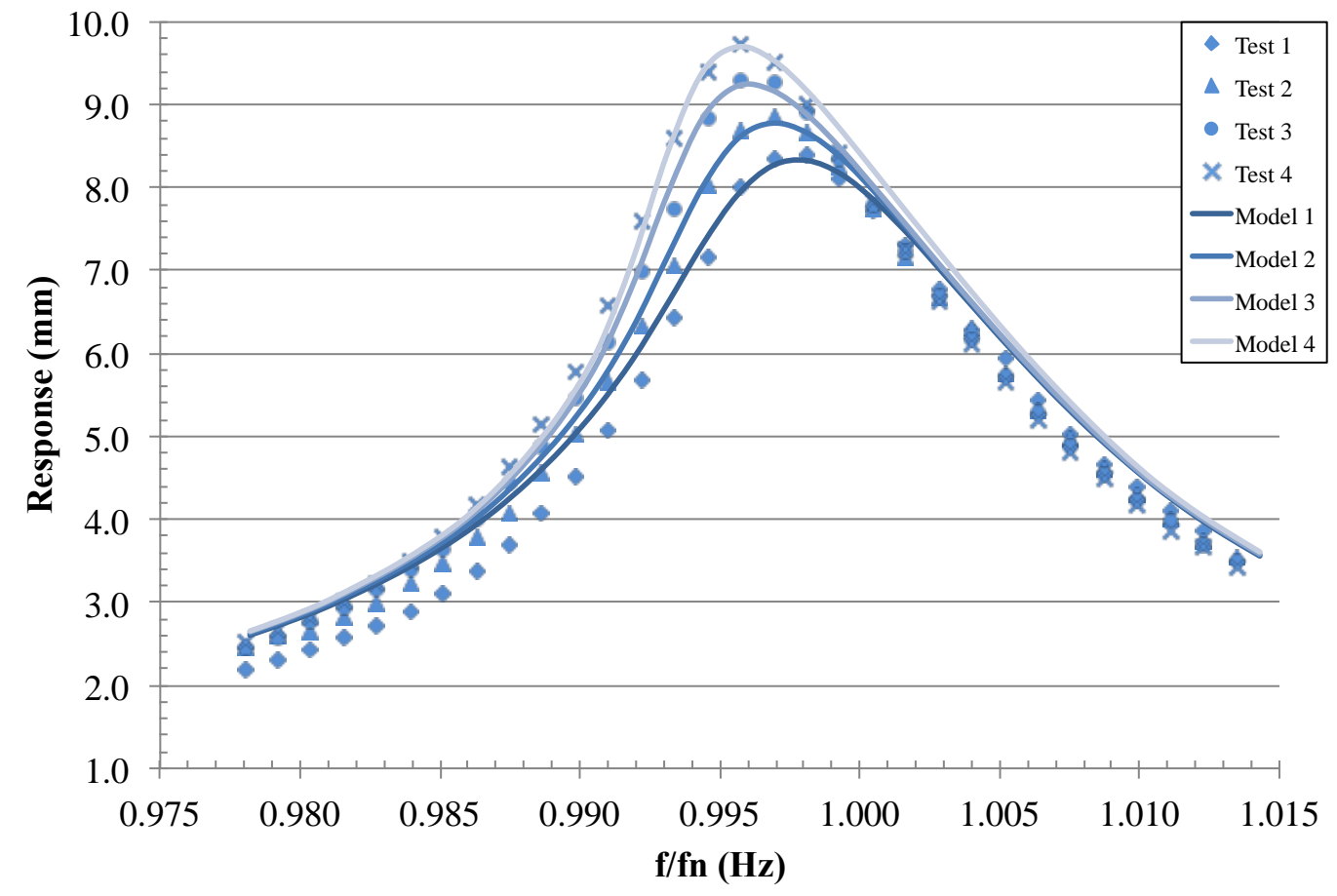

Figure 7. Experimental (markers) and analytical (solid-lines) nonlinear response for Case III 


\section{Results and Discussion}

The results of the dynamic model showed good agreement with the experimental results due to the utilization of nonlinearities in the equation of motion. This section also illustrates the link between micromechanics and the global nonlinear response, which is a powerful approach in detecting fatigue damage precursors.

\subsection{Results of Nonlinear Vibration Tests}

Each experimental set (Cases I, II, and III) contained four to five forward-step-dwell tests (Error! Reference source not found.; Figures 5-7). The beam tip response vs. the normalized excitation frequency (f/fn, where $\mathrm{f}$ and $\mathrm{fn}$ are the excitation and fundamental natural frequencies, respectively) is plotted for all cases in Figures 5-7. The objective of conducting multiple tests for each case was to capture the shift in the fundamental frequency and the increase in the response amplitude due to repeated dwells, which were symptomatic of the localized material softening effect. Figures 5-7 also show that the resonance frequency continued to drop every time the test was repeated due to the ongoing buildup of fatigue damage. The nonlinear softening effect on the resonance frequency was apparent in the increase in the beam tip response amplitude, as shown in Figures 5-7. The frequency-response curves were skewed to the left, indicating a softening nonlinearity due to a material change that eventually surpassed the structural nonlinear geometric stiffening and the gyroscopic stiffening. For all cases, the accumulation of loading cycles led to continual fatigue damage buildup, resulting in a reduction in the local stiffness manifested by a corresponding increase in the maximum beam tip deflection. 


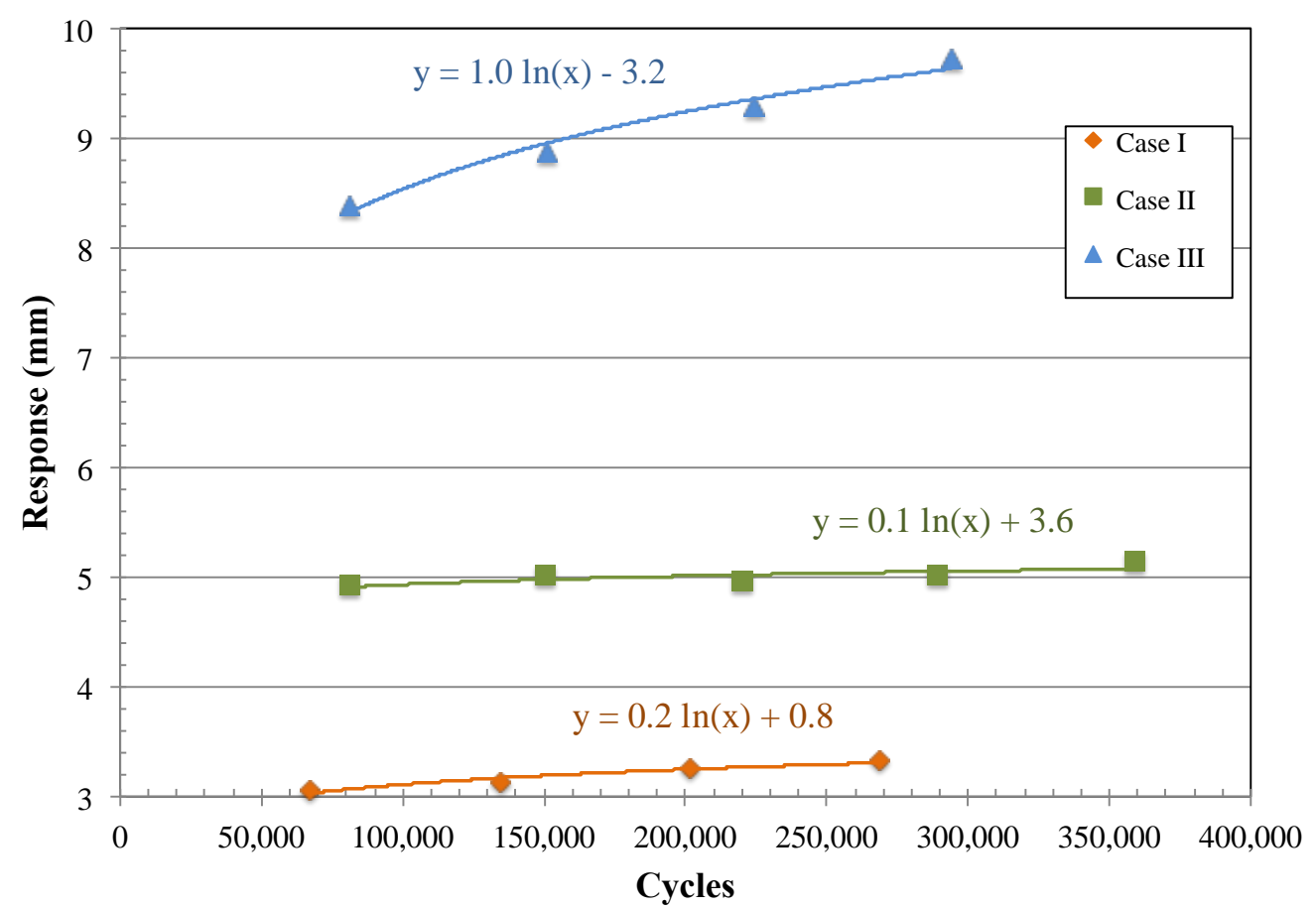

Figure 8. Maximum tip displacement for each test, as a function of number of fatigue cycles Malatkar [11] encountered similar softening phenomena in a cantilever beam exposed to transverse nonlinear base excitations. Malatkar avoided fatigue damage due to high amplitude loading by reducing the number of dwells and repeating each experiment only twice. The authors' observation of the softening behavior at the fundamental mode is similar to the softening response seen in harvester piezoceramics investigations [42-44]; however, these studies did not explain the cause of the softening. Stanton et al. [45] assumed that the nonlinear softening was due to material nonlinearities in their piezoceramic device. Softening trends were also witnessed by Saavedra and Cuitino in their study on a cracked mild steel beam exposed to harmonic vibratory loads [46]. Villanueva et al. encountered the softening response in the first mode of a nano-cantilever beam, where they assumed residual stresses due to the fabrication process as a possible instigator [47].

To verify the potential damage accumulation in the current study, nano-indentation tests were performed on a similarly-fatigued beam near the fixed boundary, which was an area of relatively 
high stress. The local elastic modulus of a sample exposed to approximately 150,000 cycles was compared to an unfatigued control sample. Figure 9 displays the results of the indentation experiments for several locations on the beam, and a minimum of 25 indents per location. The zero point indicates the clamped edge during cantilever base excitation, which was the expected position of maximum stress. The indentation elastic modulus is shown as a function of position from clamped beam location ( $x=0$ in Figure 9$)$. The horizontal dotted line in Figure 9 shows the average indentation modulus for the unfatigued sample ( 0 cycle). Indentation results for the fatigued specimen showed a clear reduction in the apparent indentation stiffness near the clamped position of the beam. The defined zero position, which included tests approximately10 $\mu \mathrm{m}$ from the fixed boundary, showed a steep drop in the apparent indentation stiffness for the fatigued sample. Stepping away from the clamped position, the mechanical response approached the behavior of the unfatigued sample. The results suggest that the average elastic response over the first few hundred nanometers of the sample surface were becoming more compliant as a result of the high number of tension-compression cycles. 


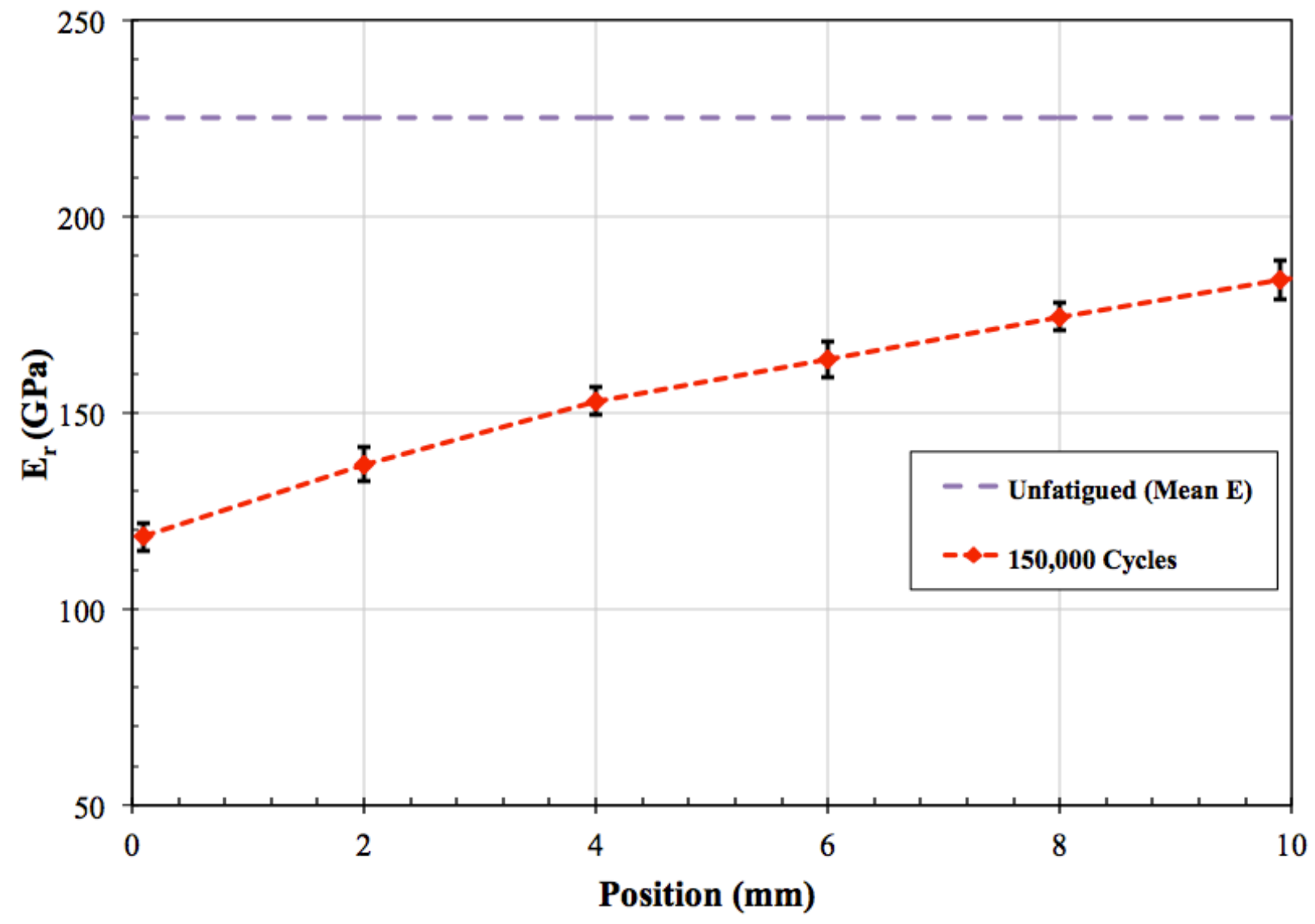

Figure 9. Indentation modulus of fatigued beam. Note that unfatigued data are average indentation results from several locations on control specimen.

To further investigate the local degradation in the material, the beam microstructure of the similarly-fatigued beam near the clamped boundary was examined via SEM. Figure 10-a shows the microstructure of a control sample, while Figure10-b shows the microstructure of the fatigued beam. For the fatigued sample, the area investigated was within $100 \mu \mathrm{m}$ of the clamped position of the beam. The control sample showed a uniform microstructure throughout, while the surface of the fatigued beam near the fixed boundary displayed a relatively high density of long dark, slender bands. The concentration of these long dark bands decreased with respect to distance from the beam clamped boundary and appeared to converge with the microstructure of the control sample. Previous investigations have indicated that fatiguing steel may cause 
formation of slip-bands, twinning, and other forms of microplasticity [49-54].
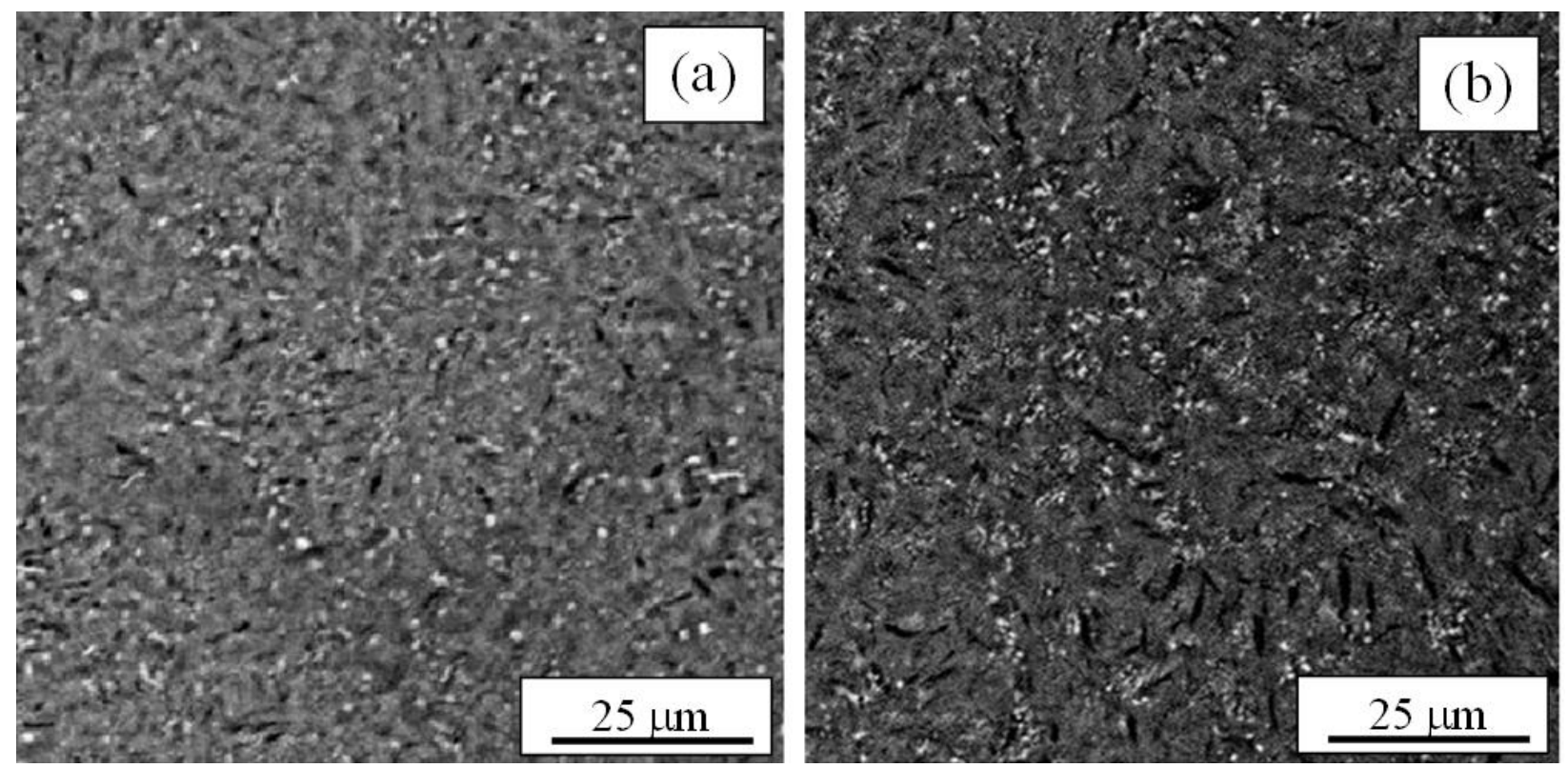

Figure 10. Microstructure of (a) unfatigued control sample with uniform microstructure and (b) fatigued specimen near clamped position (high stress location)

The indentation tests cannot fully describe variations in the dynamic behavior of the beam, but are an important first step in elucidating how cyclic fatigue damage accumulation may affect surface microstructure and thus local mechanical properties in areas of high stress concentrations. It is important to point out that for the experimental case with the highest tip displacement (Case III), the approximate maximum strain near the root was $0.8 \times 10^{-3}$ strain, which is still in the linear region of the stress-strain curve for 1095 steel. Thus, conventional linear models and sensing techniques are not sensitive enough to detect damage precursors. Alternatively, the combination of 1) the shift in the nonlinear response of the beam, and 2) the compliance effect measured through instrumented indentation ${ }_{2}$ provided a reliable identification of early stages of microplasticity, which is a known precursor to microcrack formation [50]. 


\subsection{Model Results}

The dynamic base excitations in Case I, II, and III produced nonlinear responses, which led to localized damage caused by local evolution in the material micromechanical properties near the clamped boundary. The application of linear dynamic theory does not account for the structural degradation; thus, it limits the accuracy of the dynamic response and the structural health. Therefore, the nonlinear dynamics analysis was modified to account for the fatigue-induced material evolution near the clamped end. Consequently, the modification to the nonlinear stiffness provided a better curve fit to the experimental results. The nonlinear equation of motion was solved numerically using the Runge-Kutta method using $10^{-4} \mathrm{~s}$ integration time steps. The integration time limits were set from 0 s to 30 s to ensure that the steady state condition was reached. The accelerometer mass was included in the model, but its contribution was negligible. The maximum response amplitudes from Figures 5-7 for Case I, II, and III (base excitations: 30, 50 , and $70 \mathrm{rad} / \mathrm{s}^{2}$, respectively) are plotted as a function of loading cycles in Figure 8 . The beam tip maximum response amplitudes increased as a result of increasing the excitation amplitudes; however, for each excitation level, the maximum response amplitudes increased logarithmically as a function of the loading cycles (Figure 8) due to the local material softening caused by the cyclic fatigue damage accumulation.

The structural softening response in the beam, observed in the nonlinear dynamic experiments as a fatigue damage-precursor, is captured in the analytic model by adjusting the nonlinear geometric stiffness term $k^{*}$ in the structural equation of motion (Eq. 21). Minor adjustments are also made to the viscous damping term, $c$, to match the response amplitude for each test. The governing equation is modified as follows: 


$$
\begin{aligned}
a_{1} \ddot{q}+a_{2}\left(q^{2} \ddot{q}\right. & \left.+q \dot{q}^{2}\right)+c \dot{q}+a_{3} \dot{\Omega}_{X} q^{2}+\left(k_{1}-h_{1} \Omega_{X}^{2}\right) q+\left(k^{*}-h_{2} \Omega_{X}^{2}\right) q^{3} \\
& =a_{4} \dot{\Omega}_{X}
\end{aligned}
$$

where, parameter $k^{*}$ is adjusted as follows:

$$
k^{*}=\delta k_{2}
$$

Here, the modified nonlinear stiffness, $k^{*}$, is the geometric stiffness, $k_{2}$, multiplied by the nonlinear adjustment factor, $\delta$. The model results for the response amplitude superimposed on the experimental data in Figures 5-7 show the adjustments made to the geometric stiffness and damping ratio terms in the model, which provided reasonable agreement between the experimental and model results. The nonlinear adjustment factor, $\delta$, for each test is plotted as a function of the total number of cycles for Case I, II, and III in Figures 11, 13, and 15, respectively. The experimental and numerical results show that the nonlinear adjustment factor logarithmically declines as the number of loading cycles increases. Furthermore, the adjustment factor has a negative sign, which is an indication of structural softening due to fatigue, even though the stresses in the beams are still in the elastic region. The logarithmic decay in the material stiffness is consistent with fatigue behavior [54]. For completeness, the damping ratios for Case I, II, and III are plotted as a function of fatigue cycles in Figures 11, 13, and 15, respectively. It can be seen that the beams displayed slight logarithmic decay in damping as a function of loading cycles. The beams may also exhibit nonlinear damping behavior, which is beyond the scope of this paper but worthy of further investigation. 


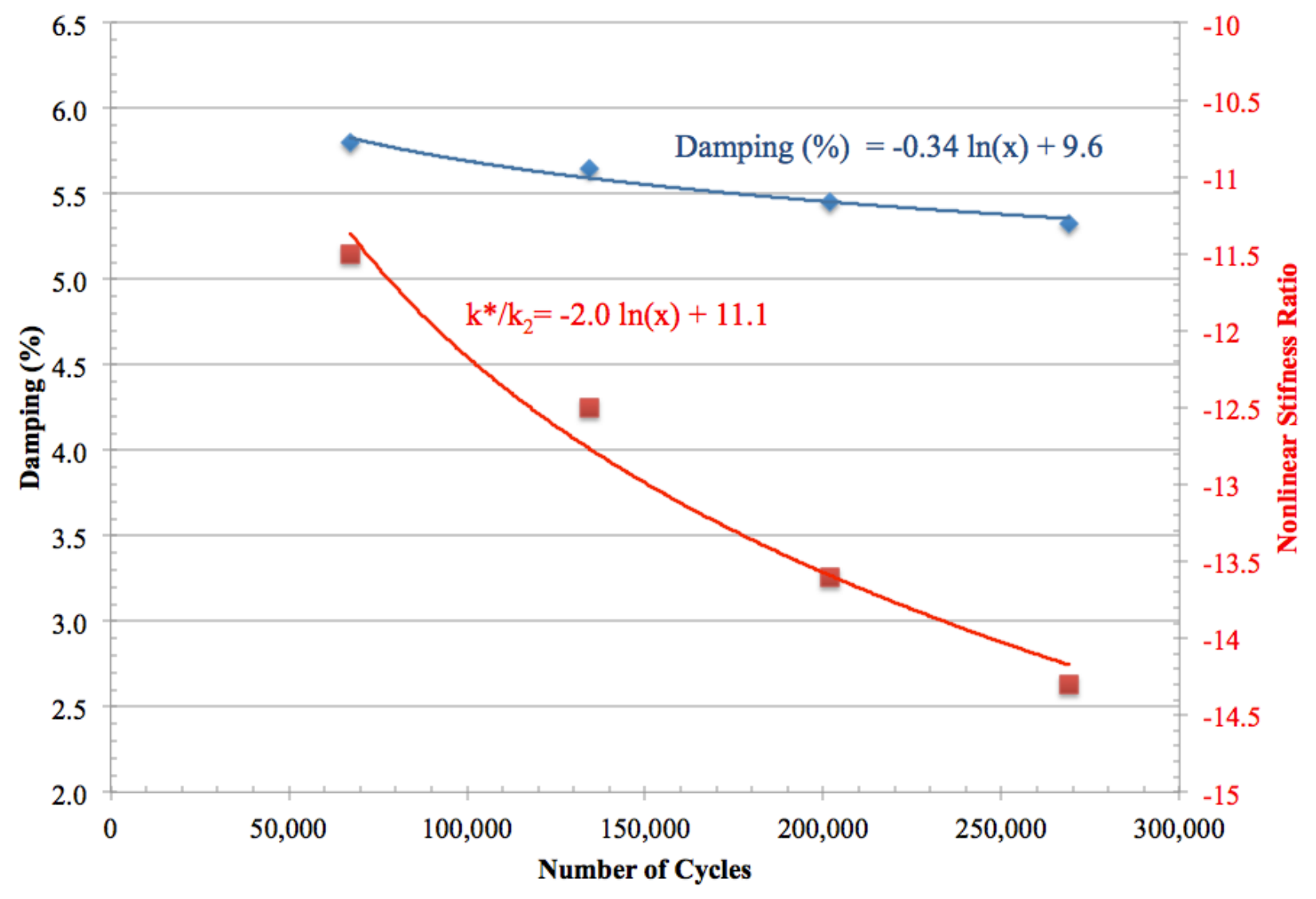

Figure 11. Nonlinear stiffness and damping adjustments as a function of cycles for Case I 


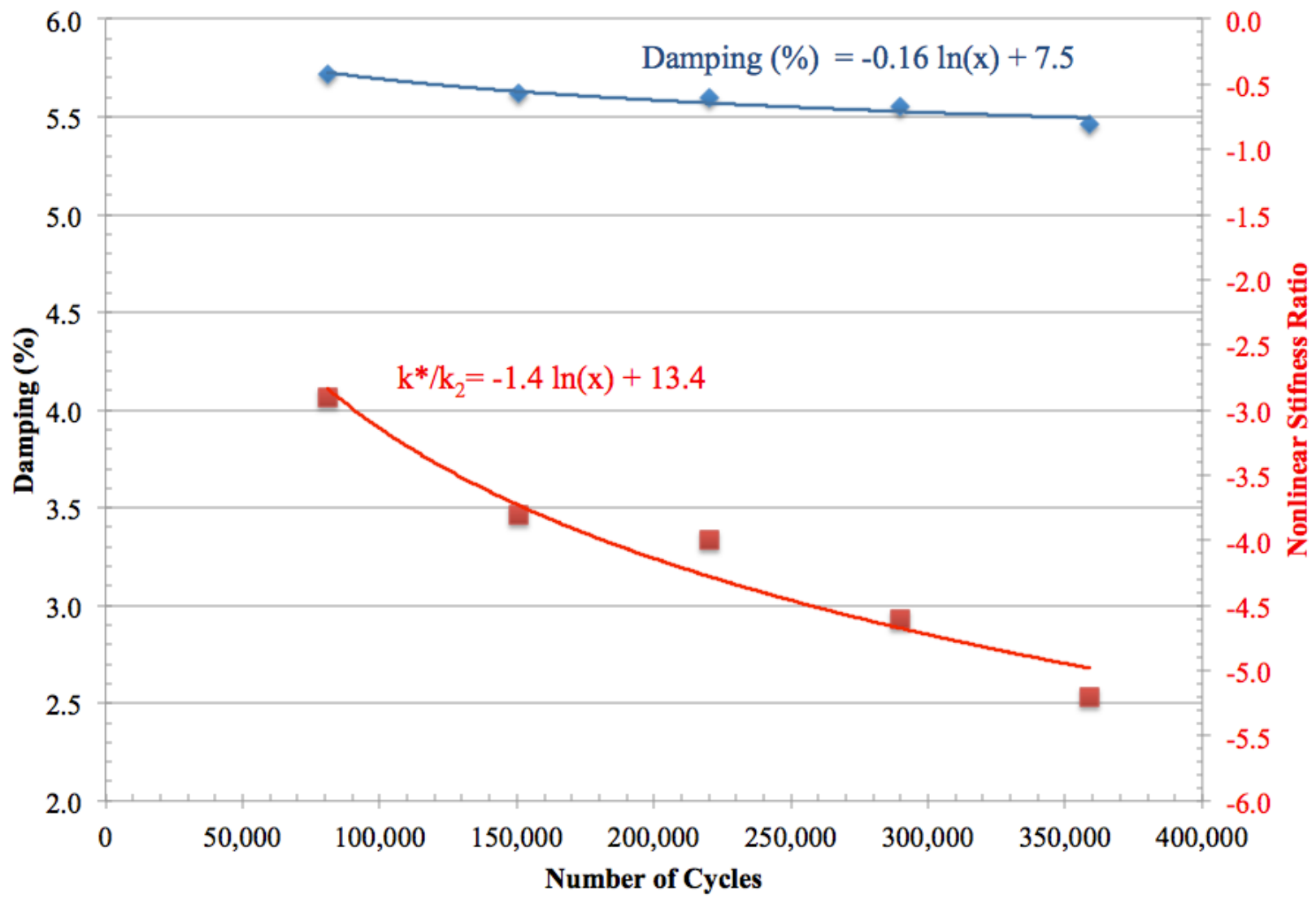

Figure 12. Nonlinear stiffness and damping adjustments as a function of cycles for Case II 


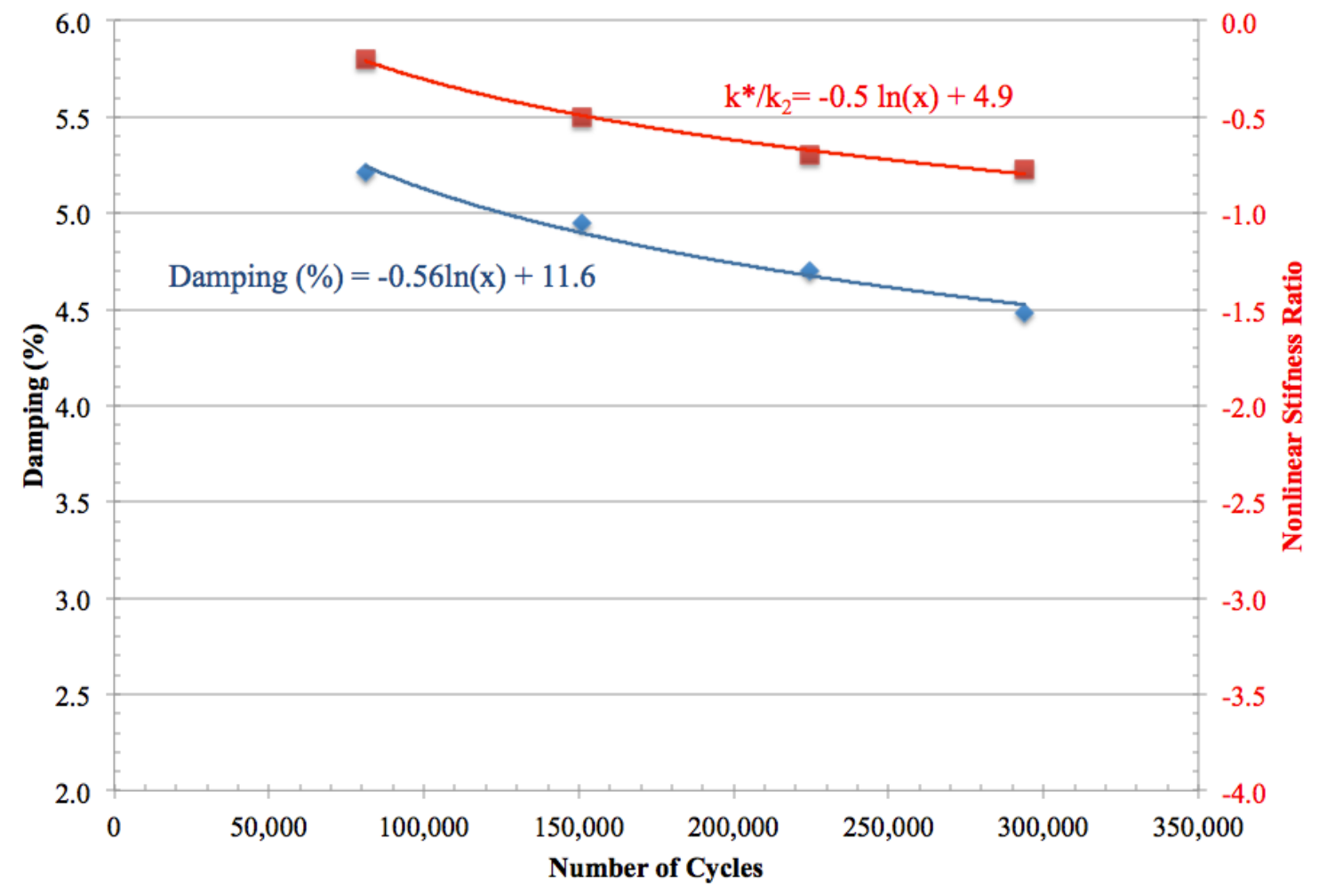

Figure 13. Nonlinear stiffness and damping adjustments as a function of cycles for Case III

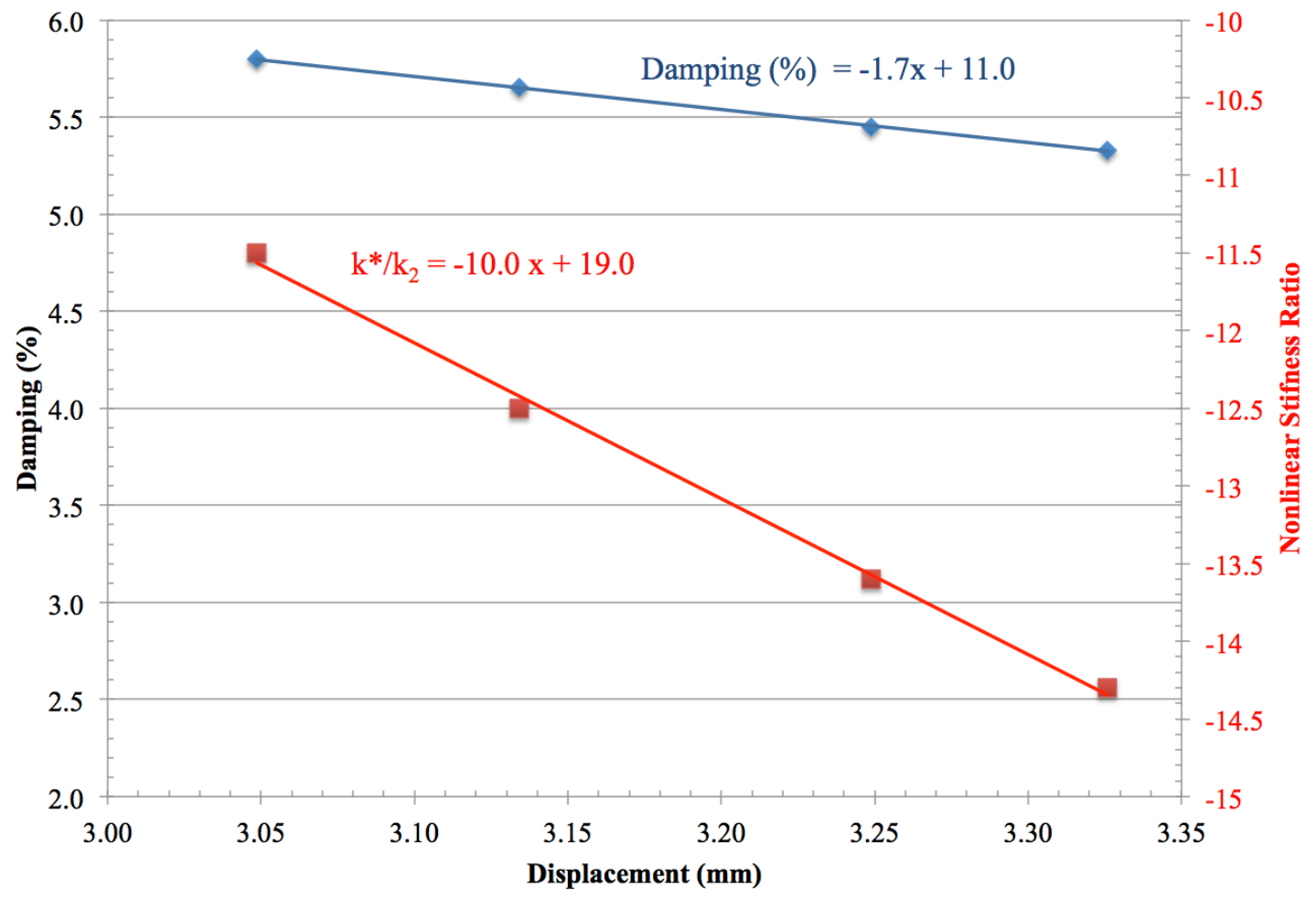

Figure 14. Nonlinear stiffness and damping adjustments as a function of displacement for Case I 


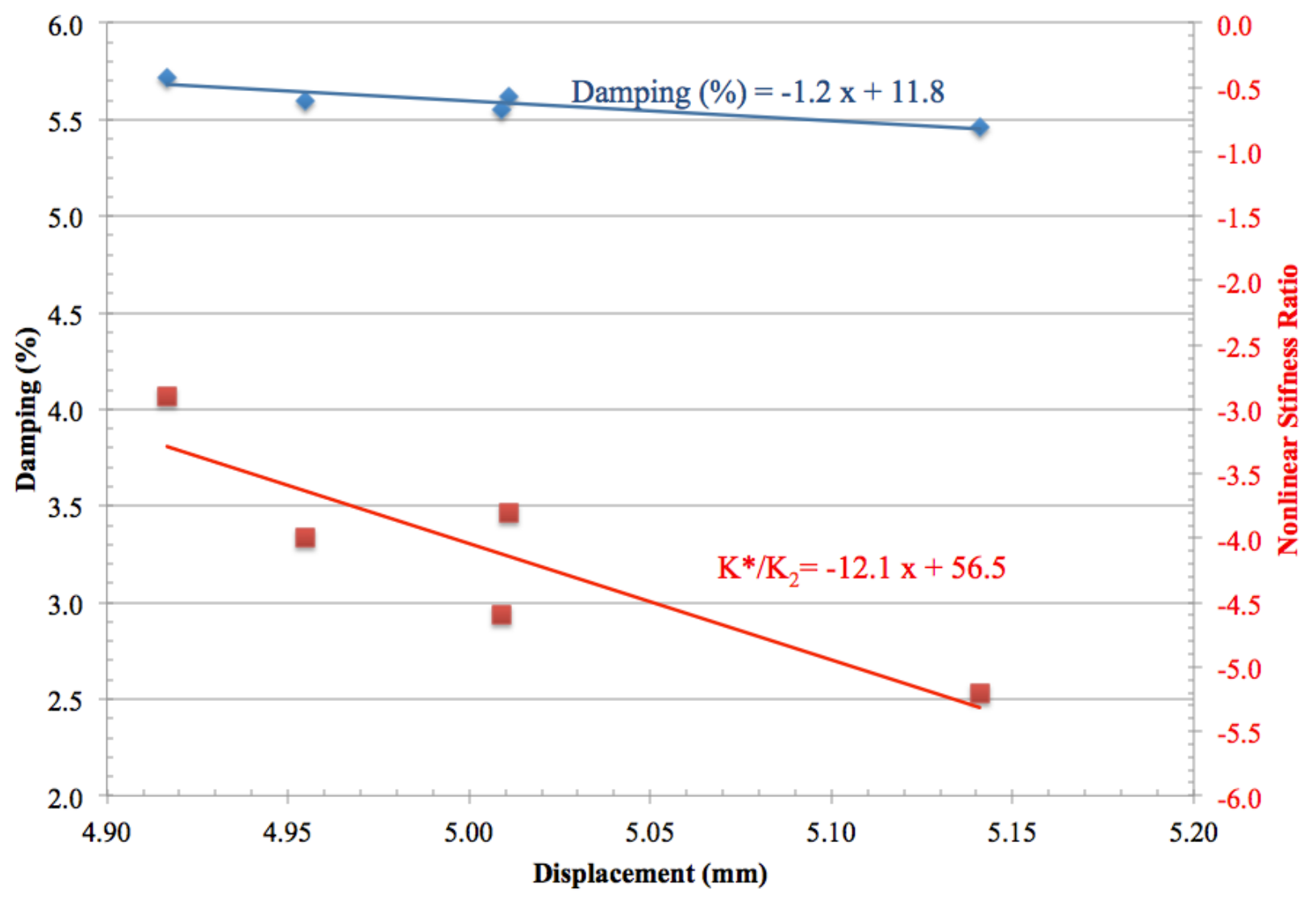

Figure 15. Nonlinear stiffness and damping adjustments as a function of displacement for Case II 


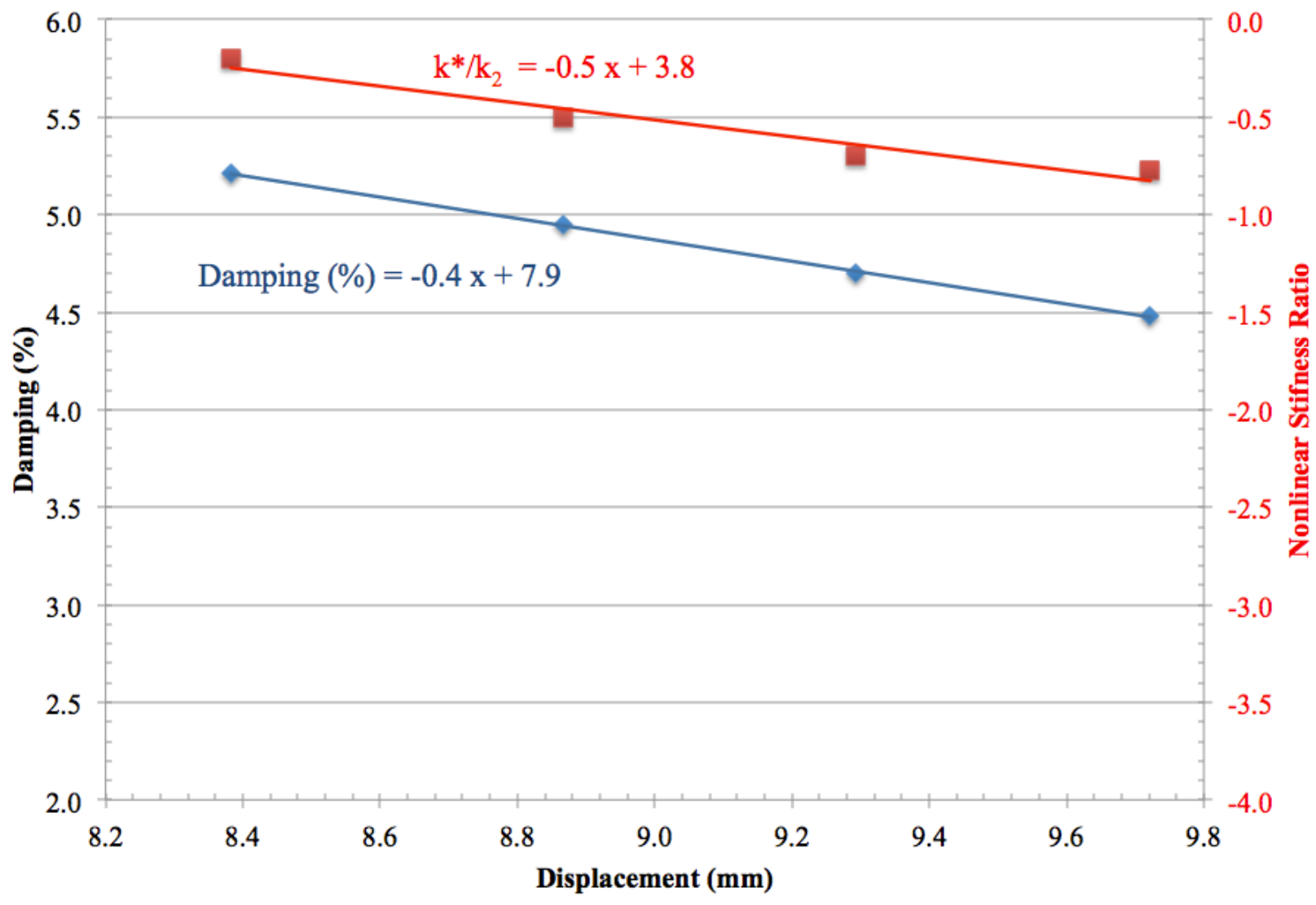

Figure 16. Nonlinear stiffness and damping adjustments as a function of displacement for Case III

It is reasonable to conclude that the local material microstructural evolution decreased the resonance frequency when the base excitation amplitude was large. The local material evolution identified in the instrumented indentation and SEM tests is likely responsible for the observed increase in the tip deflection. Therefore, it is necessary to include the evolution in the nonlinear stiffness as a function of the beam response amplitude for each excitation. Indeed, the drop in the nonlinear stiffness of the beam grew linearly with an increase in the response amplitude, as shown in Figures 12, 14, and 16. This is because the beams experienced three competing mechanisms simultaneously: 1) stiffening effect due to nonlinear geometric and kinematics effects at high amplitude response, 2) nonlinear softening due to inertia, and 3) structural 
softening effect caused by material microstructural evolution due to fatigue damage precursor. These three mechanisms subsequently increased with the increase in the base excitations, beam dynamic response, and fatigue cycles. The change in the damping ratio as a function of displacement is illustrated in Figures 12,14, and 16. The linear decrease in the damping with an increase in the beam tip displacement was minor.

The dynamic trends were consistent in all cases with the exception of Case II, where the resonance frequency in test 2,3 and 4 remained unchanged at approximately $42.10 \mathrm{~Hz}$; the resonance frequency decreased in test 5 . It is believed that the competing mechanisms between the stiffening effect (due to high response amplitude influence on geometric and gyroscopic stiffness) and the softening effect (due to nonlinear inertia term and gyroscopic softening) caused this deviation. Eventually, the material softening (decrease in the apparent stiffness) overcomes the nonlinear dynamic stiffening effect due to the continual fatigue damage. Accordingly, the beam tip response continued to increase while the resonance frequency decreased.

It is important to point out the intent of this study is to provide a first step toward incorporating material softening into the nonlinear dynamic structural equation of the beam as a potential indicator for damage precursors. Future studies would also include the position-dependent elastic modulus information into the equation of motion. Furthermore, additional investigations are needed to study the effect of other parameters on the nonlinear adjustment factor, such as variations in the: displacement amplitude, excitation frequency, boundary conditions, and multiaxial vibration loads.

\section{Summary and Conclusions}

This work describes a new approach for the detection of vibration fatigue damage precursors when a structure is subjected to rotational base excitation. The damage precursor occurred prior 
to any crack initiation in a high carbon steel structure. Instrumented indentation was used to show a local compliance effect in regions subject to relatively high stress. Nonlinear terms due to the coupling effect of the primary and secondary motions were included in the equation of motion. Exploiting the sensitivity of the nonlinear geometric stiffness enabled the detection of fatigue damage precursor. The nonlinear dynamic model provided a methodology for estimating the nonlinear dynamic response due to local material degradation prior to crack initiation, which was accomplished by adjusting the nonlinear geometric stiffness term in the equation of motion. The effect of viscous damping was also studied and found to be insignificant when compared to the geometric stiffness term. There were three opposing mechanisms that influenced the beam response: 1) stiffening due to high beam tip response amplitude and gyroscopic motion, 2) softening due to inertial forces and gyroscopic load, and 3) softening due to localized microscopic materials damage precursor. It is important to note that linear vibration-based detection would have not captured the damage precursor. Thus, the power of the nonlinear dynamic methodology for detecting damage precursor makes it a viable alternative to current inspection methods. The attraction of the nonlinear approach is that conventional sensors, such as accelerometers, are used. Further improvement of this approach could lead to a damage detection method that is both faster and less expensive than current testing techniques.

\section{Acknowledgements:}

This research effort was funded by the sponsors of the Center for Advanced Life Cycle Engineering (CALCE) at the University of Maryland and was further supported by a Collaborative Research and Development Agreement (CRADA) between the U.S. Army Research Laboratory (ARL) and the University of Maryland under ARL Open-Campus Initiative. 


\section{References:}

[1] Habtour, E., W. Connon, M. F. Pohland, S. C. Stanton, M. Paulus, and A. Dasgupta. "Review of Multiaxial Vibration in Linear and Nonlinear Structures." Shock and Vibration 2014 (2014): DOI: $10.1155 / 2014 / 294271$.

[2] Ernst, M., E. Habtour, A. Dasgupta, M. Pohland, M. Robeson, and M. Paulus. "Comparison of Electronic Component Durability under Uniaxial and Multiaxial Random Vibrations." Journal of Electronics Packaging 137.1 (2015): DOI: 10.1115/1.4028516.

[3] Chary, G. V., E. Habtour, and G. S. Drake. "Improving the Reliability in the Next Generation of US Army Platforms through Physics of Failure Analysis." Journal of Failure Analysis and Prevention 12.1 (2012): 74-85.

[4] Habtour, E., C. Choi, M. Osterman, and A. Dasgupta. "Novel Approach to Improve Electronics Reliability in the Next Generation of US Army Small Unmanned Ground Vehicles Under Complex Vibration Conditions." Journal of Failure Analysis and Prevention 12.1 (2012): 86-95.

[5] Habtour, E., D. Cole, V. Weiss, M. Robeson, R. Sridharan, and A. Dasgupta. "Detection of Fatigue Damage Precursor Using a Nonlinear Vibration Approach.” Structural Control and Health Monitoring (2016) DOI: 10.1002/stc.1844.

[6] Hodges, D. H., and E. H. Dowell. Nonlinear Equations of Motion for the Elastic Bending and Torsion of Twisted Nonuniform Rotor Blades. Rep. no. NASA TN D-7818: NASA Technical Notes, 1974.

[7] Crespo Da Silva, M. R. M., and C. C. Glynn. "Nonlinear Flexural-Flexural-Torsional Dynamics of Inextensional Beams. I. Equations of Motion." Journal of Structural Mechanic 6.4 (1978): 437-48. 
[8] Dowell, E. "Damping in Beams and Plates Due to Slipping at the Support Boundaries." Journal of Sound and Vibration 105.2 (1986): 243-53.

[9] Pai, P. F., and A. H. Nayfeh. "Non-linear Non-planar Oscillations of a Cantilever Beam under Lateral Base Excitations." International Journal of Non-Linear Mechanics 25.5 (1990): 455-74.

[10] Crespo Da Silva, M. R. M. and C. L. Zaretzky. "Nonlinear Flexural-Flexural-Torsional Interactions in Beams Including the Effect of Torsional Dynamics. I: Primary Resonance." Nonlinear Dynamics 5.1 (1994): 3-23.

[11] Malatkar, P. Nonlinear Vibrations of Cantilever Beams and Plates. Diss. Virginia Polytechnic Institute and State University, 2003. Blacksburg, VA.

[12] Glaz, B., Friedmann, P. P., and Liu, L. "Vibration Reduction and Performance Enhancement of Helicopter Rotors Using an Active/Passive Approach." AIAA Paper 2008-2178, Proceedings of the $49^{\text {th }}$ AIAA/ASME/ ASCHE/AHS/ASC Structures, Structural Dynamics \& Materials Conference, Schaumburg, IL, April 2008.

[13] Cartmell, M. P., and J. W. Roberts. "Simultaneous Combination Resonances in a Parametrically Excited Cantilever Beam." Strain 23.3 (1987): 117-26.

[14] Balachandran, B., and A. H. Nayfeh. "Nonlinear Motions of Beam-Mass Structure." Nonlinear Dynamics 1.1 (1990): 39-61.

[15] Balachandran, B., and A. H. Nayfeh. "Observations of Modal Interactions in Resonantly Forced Beam-Mass Structures." Nonlinear Dynamics 2.2 (1991): 77-117.

[16] Jaworski, J. W., and E. H. Dowell. "Free Vibration of a Cantilevered Beam with Multiple Steps: Comparison of Several Theoretical Methods with Experiment." Journal of Sound and Vibration 312.4-5 (2008): 713-25. 
[17] Ansari, M., E. Esmailzadeh, and N. Jalili. "Exact Frequency Analysis of a Rotating Cantilever Beam With Tip Mass Subjected to Torsional-Bending Vibrations." Journal of Vibration and Acoustics 133.4 (2011).

[18] Kane, T. R., R. Ryan, and A. K. Banerjee. "Dynamics of a Cantilever Beam Attached to a Moving Base." Journal of Guidance, Control, and Dynamics 10.2 (1987): 139-51.

[19] Yoo, H. H., R. R. Ryan, and R. A. Scott. "Dynamics of Flexible Beams Undergoing Overall Motions." Journal of Sound and Vibration 181.2 (1995): 261-78.

[20] Glaz, B., Friedmann, P. P., Liu, L., Kumar, D., and Cesnik, C. E. S. "The AVINOR Aeroelastic Simulation Code and its Application to Reduced Vibration Composite Rotor Blade Design." AIAA Paper 2009-2601, Proceedings of the 50 ${ }^{\text {th }}$ AIAA/ASME/ASCHE/AHS/ASC Structures, Structural Dynamics \& Materials Conference, Palm Springs, CA, May 2009. [21] Bauchau, O. A., and C. H. Hong. "Finite Element Approach to Rotor Blade Modeling." Journal of the American Helicopter Society 32.1 (1987): 60-67.

[22] Hodges, D. H. "Review of Composite Rotor Blade Modeling," AIAA Journal, 3.82, (1990): 561-65.

[23] Yigit, A., R. Scott, and A. Galipulsoy. "Flexural Motion of a Radially Rotating Beam Attached to a Rigid Body." Journal of Sound and Vibration 121.2 (1988): 201-10.

[24] Liao, C., and Y. Y. Dang. "Structural Characteristics of Spinning Pretwisted Orthotropic Beams." Computers \& Structures 45.4 (1992): 715-31.

[25] Chen, W. R. "On the Vibration and Stability of Spinning Axially Loaded Pre-twisted Timoshenko Beams." Finite Elements in Analysis and Design 46.11 (2010): 1037-47.

[26] Smith, C., and H. Baruh. "Dominance of Stiffening Effects for Rotating Flexible Beams." Journal of Guidance, Control, and Dynamics 14.5 (1991): 1072-74. 
[27] Anderson, T.J., B. Balachandran and A. H. Nayfeh. "Nonlinear Resonances in a Flexible Cantilever Beam.” Journal of Vibration and Acoustics 116.4 (1994): 480-84.

[28] Baruh, H. Analytical Dynamics. Boston, MA: WCB/McGraw-Hill, 1999.

[29] Meirovitch, L. Fundamentals of Vibrations. Boston: McGraw-Hill, 2001.

[30] Hamdan, M. N., and M. H. F. Dado. "Large Amplitude Free Vibrations Of A Uniform Cantilever Beam Carrying An Intermediate Lumped Mass And Rotary Inertia." Journal of Sound and Vibration 206.2 (1997): 151-68.

[31] Elvin, N. G., and A. A. Elvin. "A General Equivalent Circuit Model for Piezoelectric Generators." Journal of Intelligent Material Systems and Structures 20.1 (2008): 3-9.

[32] Leadenham, S., and A. Erturk. "Unified Nonlinear Electroelastic Dynamics of a Bimorph Piezoelectric Cantilever for Energy Harvesting, Sensing, and Actuation." Nonlinear Dynamics 79.3 (2014): 1727-43.

[33] Nayfeh, A. H. and D. T. Mook, Nonlinear Oscillations, Wiley-VCH, New York, 1995.

[34] Oliver, W. C., and G. M. Pharr. “An Improved Technique for Determining Hardness and Elastic Modulus Using Load and Displacement Sensing Indentation Experiments." Journal of Materials Research 7.6 (1992): 1564-83.

[35] Saha, R., and W. D. Nix. "Effects of the Substrate on the Determination of Thin Film Mechanical Properties by Nanoindentation.” Acta Materialia 50 (2002): 23-38.

[36] Cole, D. P., H. Jin, W. Lu, A. L. Roytburd, and H. A. Bruck. "Reversible Nanoscale Deformation in Compositionally Graded Shape Memory Alloy Films.” Applied Physics Letters 94 (2009): 193114.

[37] Cole, D. P., H. A. Bruck, A. L. Roytburd. "Nanomechanical Characterisation of Graded NiTi Films Fabricated Through Diffusion Modification,” Strain 45 (2009): 232-37. 
[38] Cole, D. P. and K. S. Strawhecker. “An Improved Instrumented Indentation Technique for Single Microfibers.” Journal of Materials Research 29.9 (2014): 1104-12.

[39] Strawhecker, K. S., and D. P. Cole. "Ballistic Fiber Surface Characterization.” Journal of Applied Polymer Science 131 (2014): 40880.

[40] Dutta, A. K., D. Penumadu, and B. Files. "Nanoindentation Testing for Evaluating Modulus and Hardness of Single-Walled Carbon Nanotube-Reinforced Epoxy Composites." Journal of Materials Research 19 (2004): 158-164.

[41] Cole, D. P., A. L. M. Reddy, M. G. Hahm, R. McCotter, A. H. C. Hart, R. Vajtai, P. M. Ajayan, S. P. Karna, and M. L. Bundy. "Electromechanical Properties of Polymer ElectrolyteBased Stretchable Supercapacitors.” Advanced Energy Materials 4 (2014): 1300844.

[42] Kacem, N., J. Arcamone, F. Perez-Murano, and S. Hentz. "Dynamic Range Enhancement of Nonlinear Nanomechanical Resonant Cantilevers for Highly Sensitive NEMS Gas/mass Sensor Applications." Journal of Micromechanics and Microengineering 20.4 (2010): 045023. [43] Priya S, D. Viehland, and A. Carazo. "High-Power Resonant Measurements of Piezoelectric Materials: Importance of Elastic Nonlinearities." Journal of Applied Physics 90.3 (2001): 146979.

[44] Yu, S., S. He, and W. Li "Theoretical and Experimental Studies of Beam Bimorph Piezoelectric Power Harvesters." Journal of Mechanics and Materials of Structures 5.3 (2010): 427-45.

[45] Stanton, S. C., A. Erturk, B. P. Mann, E. H. Dowell, and D. J. Inman. "Nonlinear Nonconservative Behavior and Modeling of Piezoelectric Energy Harvesters Including Proof Mass Effects,” Journal of Intelligent Material Systems and Structures 23.2 (2012): 183-199. [46] Saavedra, P. N., and L. A. Cuitino. "Crack Detection and Vibration Behavior of Cracked 
Beams." Computers \& Structures 79.16 (2001): 1451-59.

[47] Villanueva, L. G., R. B. Karabalin, M. H. Matheny, D. Chi, J. E. Sader, and M. L. Roukes. "Nonlinearity in Nanomechanical Cantilevers." Physical Review B 87 (2013).

[48] Villarino de Castro, D. B., J. M. Ventura, C. O. F. T. Ruckert, D. Spinelli, W. W. B. Filho. "Influence of Phosphorus Content and Quenching/Tempering Temperature on Fracture Toughness and Fatigue Life of SAE 5160 Steel.” Materials Research 13(4) (2010): 445-55.

[49] Kang, S.K., Y-S. Jung, B-G. Yoo, J-I. Jang, and Y-K. Lee. "Orientation-Dependent Indentation Modulus and Yielding in a High Mn Twinning-Induced Plasticity Steel." Materials Science and Engineering A 532 (2012): 500-04.

[50] Sangid, M. D. "The Physics of Fatigue Crack Initiation." International Journal of Fatigue 57 (2013): 58-72.

[51] Mura, T. Micromechanics of Defects in Solids. The Hague: Nijhoff, 1982.

[52] Charsley, P., and M.P.E. Desvaux. "The Behaviour of Copper-12\% Aluminium under Simple Reversed Stresses." Materials Science and Engineering 4.4 (1969): 211-20.

[53] Tanaka, K., and T. Mura. "A Theory of Fatigue Crack Initiation at Inclusions." Metallurgical Transactions A 13.1 (1982): 117-23

[54] Dowling, N. E., Mechanical Behavior of Materials: Engineering Methods for Deformation, Fracture, and Fatigue, Prentice Hall, Upper Saddle River, NJ, USA, 2nd edition, 1999. 